\title{
Note on multiplicities of ideals
}

\author{
By Christer Lech
}

\section{Introduction}

In this note we prove some formulae involving lengths and multiplicities of ideals. Our results are incomplete but, in the absence of more final results, they are perhaps not without interest.

We begin by proving a generalization of Samuel's well-known inequality $e\left(x_{1}, \ldots, x_{r}\right) \leqslant L\left(x_{1}, \ldots, x_{r}\right)$ interrelating the multiplicity and length of an ideal generated by a system of parameters in a local ring (Theorem 1). By combining this generalization with an argument in [2] we obtain an asymptotic expression for $e\left(x_{1}, \ldots, x_{r}\right)$ which is more general than the one given in the paper cited (Theorem 2).

The rest of the note is independent of the results just mentioned and mainly concerns flat couples of local rings (Serre, [10], pp. 34-41). Let $\left(Q_{0}, Q\right)$ be such a couple with maximal ideals $\left(\mathrm{m}_{0}, \mathfrak{m}\right)$. Assume that $\mathrm{m}_{0} Q$ is a $\mathrm{m}$-primary ideal or, equivalently, that $Q_{0}$ and $Q$ have the same dimension. Denote by $e\left(Q_{0}\right)$ and $e(Q)$ the multiplicities of $m_{0}$ and $m$ respectively. We prove that if the dimension of $Q_{0}$ and $Q$ is less than or equal to two, then

$$
e\left(Q_{0}\right) \leqslant e(Q),
$$

and we make some further observations in support of a conjecture that this inequality is always true. The truth of the conjecture would imply the general truth of the inequality

$$
e\left(Q_{\mathfrak{p}}\right) \leqslant e(Q)
$$

for prime ideals $\mathfrak{p}$ of $Q$ satisfying $\operatorname{dim} \mathfrak{p}+\operatorname{rank} \mathfrak{p}=\operatorname{dim} Q$. For, according to Nagata ([4], $\S 13)$, this inequality is valid when $Q$ is complete, and one could pass from $Q$ to its completion $Q^{*}$ by means of a suitable flat couple $\left(Q_{\mathfrak{p}}, Q_{\mathfrak{p}^{*}}^{*}\right)$. - However, our arguments are powerless in the general case. The result for dimension two is obtained by using ideals similar to form ideals but generated by power products of the variables. An application of these ideals also gives another estimate which bears a slight resemblance to the formula $e\left(Q_{p}\right) \leqslant e(Q)$ (Theorem 3).

Serre defines flat couples in homological terms. In the present note we use hardly anything of the homological machinery, and in an appendix we give an alternative non-homological definition of flat couples, which would serve us equally well and which ties up this new concept with an older result of SamuelNagata. 


\section{Preliminaries}

In this section we settle some questions of terminology and notation, and recall some prerequisites. (As a general reference for the terminology, see Northcott's book [5]; concerning length and multiplicity, see e.g. [2].)

(i) Let $F\left[\bar{X}_{1}, \ldots, X_{r}\right]$ be a ring of formal polynomials over a field $F$. An ideal in $F\left[X_{1}, \ldots, X_{r}\right]$ will be called multihomogeneous if it can be generated by elements of the form $X_{1}^{n_{1}} \ldots X_{r}^{n_{r}}$. This agrees with the customary use of the term, except that we have settled, once for all, a particular division of the set of variables, namely the one where each subset contains just one variable. Every multihomogeneous ideal has a normal decomposition in which the primary components are also multihomogeneous. This or $n$ be shown by replacing, in a given decomposition, each component by the largest multihomogeneous ideal contained in it.

(ii) If $\mathfrak{q}$ is a primary ideal of a Noetherian ring $R$, we denote its length by $L(\mathfrak{q})$ and its multiplicity by $e(\mathfrak{q})$; if $\mathfrak{a}$ is an ideal in $R$ and $\mathfrak{p}$ a minimal prime ideal of $\mathfrak{a}$, we put $L(\mathfrak{a} ; \mathfrak{p})=L\left(\mathfrak{a} R_{\mathfrak{p}}\right)$ and $e(\mathfrak{a} ; \mathfrak{p})=e\left(\mathfrak{a} R_{\mathfrak{p}}\right)$, where $R_{\mathfrak{p}}$ denotes the generalized ring of quotients with respect to $\mathfrak{p}$; if $Q$ is a local ring, $e(Q)$ will denote the multiplicity of its maximal ideal. Suppose that $q=\left(q_{1}, \ldots, q_{r}\right)$. Then we shall use $L\left(q_{1}, \ldots, q_{r}\right)$ as an alternative of $L(q)$, and similarly for other functions of ideals. In Section 2 we shall further use $L(a, \alpha)$ to denote the length of an ideal $(\mathfrak{a}, \alpha)$ generated by an ideal $\mathfrak{a}$ and an element $\alpha$.

(iii) We recall the associativity formula for multiplicities ([2], [4]). Let $\left\{x_{1}, \ldots, x_{r}\right\}$ be a system of parameters in a local ring. Put $\mathfrak{a}=\left(x_{1}, \ldots, x_{m}\right), \mathfrak{b}=\left(x_{m+1}, \ldots, x_{r}\right)$ $(0 \leqslant m \leqslant r)$. Then

$$
e(\mathfrak{a}+\mathfrak{b})=\sum_{\mathfrak{p}} e((\mathfrak{a}+\mathfrak{p}) / \mathfrak{p}) e(\mathfrak{b} ; \mathfrak{p}),
$$

where $\mathfrak{p}$ ranges over those minimal prime ideals of $\mathfrak{b}$ for which $\operatorname{dim} \mathfrak{p}+\operatorname{rank} \mathfrak{p}=r$. As a corollary we have

(See [2], p. 314.)

$$
e\left(x_{1}^{n_{1}}, \ldots, x_{r}^{n_{r}}\right)=n_{1} \ldots n_{r} e\left(x_{1}, \ldots, x_{r}\right) .
$$

We shall also use another formula, relat?d to the associativity formula. Let $Q$ be a local ring and lei $q$ ic a primary dieal bolonging to the maximal ileal of $Q$. Let $\mathfrak{p}_{1}, \ldots, \mathfrak{p}_{s}$ be those prime ideals in $Q$ whose dimensions are equal to $\operatorname{dim} Q$, and let $n_{1}, \ldots, n_{s}$ be the corresponding primary components of the zero ideal. Then

$$
e(\mathfrak{q})=\sum_{i=1}^{s} e\left(\left(\mathfrak{q}+\mathfrak{p}_{i}\right) / \mathfrak{p}_{i}\right) L\left(\mathfrak{n}_{i}\right)
$$

We shall call this formula the additivity formula. A short proof can be obtained by using a lemma by Artin-Rees (cf. [2], the addendum; see also e.g. [2], Section 4).

(iv) Let $Q$ be a local ring of dimension $r$ and let $\left\{x_{1}, \ldots, x_{r}\right\}$ be a system of parameters in $Q$. Denote by $m$ the maximal ideal of $Q$. In the deduction of Theorem 2 from Theorem 1 , and also in Section 5, we shall apply a formula in [2] (the formula (6), p. 305) which gives an expression for the length of an 
arbitrary m-primary ideal in $Q$. If we let $\mathfrak{q}$ denote such an ideal, the formula can be written as follows:

$$
L(\mathfrak{q})=\sum_{\nu=0}^{l-1} L\left(I_{\nu}(\mathfrak{q})\right)
$$

It refers to an arbitrary but fixed composition series of the $Q$-module $Q /\left(x_{1}, \ldots, x_{r}\right)$. The values of $\nu$ involved correspond to the successive steps of this series; in particular, $l=L\left(x_{1}, \ldots, x_{r}\right)$. The $I_{v}(q)$ are homogeneous, $\left(X_{1}, \ldots, X_{r}\right)$-primary ideals of the polynomial $\operatorname{ring} K[X]=K\left[X_{1}, \ldots, X_{r}\right]$ where $K=Q / \mathrm{m}$. In fact, with each ideal $\mathfrak{a}$ in $Q$ there are associated $l$ homogeneous ideals $I_{v}(\mathfrak{a})(\nu=0,1, \ldots, l-1)$ in $K[X]$, which enjoy the following properties ${ }^{1}$ :

(A) $\mathfrak{a} \subseteq \mathfrak{b}$ implies $I_{v}(\mathfrak{a}) \subseteq I_{\nu}(\mathfrak{b})$;

(B) $I_{v}(0)=(0)$ for exactly $e\left(x_{1}, \ldots, x_{r}\right)$ values of $v$;

(C) $I_{0}(\mathfrak{a}) \subseteq I_{v}(\mathfrak{a}) \quad(v=0,1, \ldots, l-1)$;

(D) If $x_{1}^{n_{2}} \ldots x_{r}^{n_{r}} \in \mathfrak{a}$, then $X_{1}^{n_{1}} \ldots X_{r}^{n_{r}} \in I_{0}(\mathfrak{a})$. Further, suppose that $Q$ contains a field $k$ (the equicharacteristic case), and let $\varphi \in k\left[x_{1}, \ldots, x_{r}\right] \cap a$. Denote by $\bar{\varphi}$ the element of $k\left[x_{1}, \ldots, x_{r}\right]$ which is equal to the sum of the terms of lowest degree in $\varphi$. Then $I_{0}(\mathfrak{a})$ contains the form that is obtained from $\bar{\varphi}$ by replacing $x_{i}$ by $X_{i}(i=1,2, \ldots, r)$ and the coefficients in $k$ by their canonical images in $K$.

These properties of the $I_{v}(\mathfrak{a})$ will underlie our applications of the formula (1.2).

(v) Consider the four conditions on a local ring $Q$ of dimension $r$ which consist in claiming the validity of the statements (A) and (B) below a) for at least one system of parameters $\left\{x_{1}, \ldots, x_{r}\right\}$ in $Q$, b) for all such systems.

$$
\begin{aligned}
& \text { (A) } e\left(x_{1}, \ldots, x_{r}\right)=L\left(x_{1}, \ldots, x_{r}\right) \text {; } \\
& \text { (B) }\left(x_{1}, \ldots, x_{m}\right): x_{m+1}=\left(x_{1}, \ldots, x_{m}\right) \quad(0 \leqslant m \leqslant r-1) .
\end{aligned}
$$

These four conditions ( $\mathrm{Aa}, \mathrm{Ab}, \mathrm{Ba}, \mathrm{Bb}$ ) are equivalent, and if they are satisfied, $Q$ is called a Cohen-Macauley ring. (The concept of Cohen-Macauley ring was introduced simultaneously in [4], [6], and [8]. We shall use it only to make the statement of Theorem 1 as complete as possible.)

\section{Generalization of the formula $e\left(x_{1}, \ldots, x_{r}\right) \leqslant L\left(x_{1}, \ldots, x_{r}\right)$}

Denote by $Q$ a local ring of dimension $r$ and let $\left\{x_{1}, \ldots, x_{r}\right\}$ be a system of parameters in $Q$. We shall be dealing with ideals in $Q$ generated by power products of $x_{1}, \ldots, x_{r}$. To get a suitable notation for these ideals we introduce a ring of formal polynomials, $F[X]=F\left[X_{1}, \ldots, X_{r}\right]$, where $F$ is a quite arbitrary field. If $I$ is a multihomogeneous ideal of $F[X]$ (or $F\left[X_{1}, \ldots, X_{r-1}\right]$ ), then $\bar{I}$ will denote the ideal in $Q$ generated by those power products $x_{1}^{n_{1}} \ldots x_{r}^{n_{r}}$ for which $X_{1}^{n_{1}} \ldots X_{r}^{n_{r}} \in I$.

1 The properties (C) and (D) are not stated explicitly in [2], but follow directly from the definitions. 
CH. LECH, Note on multiplicities of ideals

Theorem 1. Let $I$ be a multihomogeneous, $\left(X_{1}, \ldots, X_{r}\right)$-primary ideal in $F[X]$. Then

$$
L(I) \geqslant L(I) e\left(x_{1}, \ldots, x_{r}\right)
$$

Equality holds if and only if $Q$ is a Cohen-Macauley ring.

For $I=\left(X_{1}, \ldots, X_{r}\right)$ this theorem gives Samuel's formula

$$
e\left(x_{1}, \ldots, x_{\tau}\right) \leqslant L\left(x_{1}, \ldots, x_{r}\right) \text {. }
$$

To prove the theorem we shall need a lemma.

Lemma 1. Let $r \geqslant 1$. Then

$$
e\left(\left(x_{1}, \ldots, x_{r}\right) /\left(x_{r}\right)\right) \geqslant e\left(x_{1}, \ldots, x_{\tau}\right) .
$$

Equality holds if and only if $x_{r}$ is not contained in any $(r-1)$-dimensional prime ideal belonging to the zero ideal of $Q$.

Proof of Lemma 1. First assume $r=1$. Put $x_{1}=x$. We have to prove that $L(x) \geqslant e(x)$, and that $L(x)=e(x)$ if and only if the maximal ideal of $Q$ does not belong to the zero ideal. This is true according to Samuel's formula cited above and by the properties of Cohen-Macauley rings. - A simple direct proof is obtained by considering the $Q$-homomorphism

$$
Q /(x) \rightarrow\left(x^{n}\right) /\left(x^{n+1}\right)
$$

induced by multiplication with $x^{n}$. This homomorphism is onto and has the kernel $\left((0): x^{n}+(x)\right) /(x)$, which varies monotonously with $n$. In view of the definition of $e(x)$ one sees that $L(x) \geqslant e(x)$, and that $L(x)=e(x)$ if and only if $(0): x^{n} \subseteq(x)$ (all $n$ ). The latter condition is easily seen to be equivalent with $(0): x^{n} \subseteq \bigcap_{v=1}^{\infty}\left(x^{v}\right)=(0)$ (all $n$ ), which gives the result.

When $r>1$, we apply the associativity formula twice: first to the ring $Q$ and the parameters $x_{1}, \ldots, x_{r}$, then to the ring $Q /\left(x_{r}\right)$ and the parameters in that ring represented by $x_{1}, \ldots, x_{r-1}$. Both times we take $m=r-1$. This gives the two formulae

$$
\begin{aligned}
& e\left(x_{1}, \ldots, x_{r}\right)=\sum_{\mathfrak{p}} e\left(\left(\left(x_{1}, \ldots, x_{r-1}\right)+\mathfrak{p}\right) / \mathfrak{p}\right) e\left(\left(x_{r}\right) ; \mathfrak{p}\right), \\
& e\left(\left(x_{1}, \ldots, x_{r}\right) /\left(x_{r}\right)\right)=\sum_{\mathfrak{p}^{\prime}} e\left(\left(\left(x_{1}, \ldots, x_{r-1}\right)+\mathfrak{p}^{\prime}\right) / \mathfrak{p}^{\prime}\right) L\left(x_{r} Q_{\mathfrak{y}^{\prime}}\right),
\end{aligned}
$$

where $\mathfrak{p}$ ranges over those minimal prime ideals of $\left(x_{r}\right)$ for which $\operatorname{dim} \mathfrak{p}=r-1$ and rank $\mathfrak{p}=1$, and where $\mathfrak{p}^{\prime}$ ranges over (all) those for which $\operatorname{dim} \mathfrak{p}^{\prime}=r-1$. The statement of the lemma follows by a comparison between the two formulae, taking the previous, one-dimensional result into account.

Remark. By an iterated use of Lemma 1 one could prove the equivalence of the four properties which caracterize Cohen-Macauley local rings (cf. Section 1, (v)). 
Proof of Theorem 1. Let us first settle one minor point. Suppose that $I$ is a multihomogeneous, $\left(X_{1}, \ldots, X_{r}\right)$-primary ideal in $F(X)$, and that. $X^{(\mu)}$ is a power product in $I:\left(X_{1}, \ldots, X_{r}\right)$ not belonging to $I$. Consider the $Q$-homomorphism

$$
Q /\left(x_{1}, \ldots, x_{r}\right) \rightarrow \overline{\left(I, X^{(\mu)}\right)} / \bar{I}
$$

induced by multiplication with the power product $x^{(\mu)}$ of $x_{1}, \ldots, x_{r}$ that corresponds to $X^{(\mu)}$. Since this homomorphism is onto, it follows that

$$
\left.L(\bar{I})-L\left(\overline{\left(I, X^{(\mu)}\right.}\right)\right) \leqslant L\left(x_{1}, \ldots, x_{r}\right)
$$

Hence, by induction, $\quad L(\bar{I}) \leqslant L(I) L\left(x_{1}, \ldots, x_{r}\right)$.

If $Q$ is a Cohen-Macauley ring, this means that

$$
L(\bar{I}) \leqslant L(I) e\left(x_{1}, \ldots, x_{r}\right) .
$$

For such a $Q$ it therefore suffices to prove the reverse inequality.

Now we come to the main part of the proof. We shall use induction, in the first place on $r$. When $r=0$, we have $I=(0)$, and the contention is trivially true. If $r=1$, then $I=\left(X_{1}^{n}\right)(n \geqslant 1)$, and the result follows from Lemma 1, applied with $r=1$. Assume then that $r \geqslant 2$ and that the theorem is true when $r$ is replaced by $r-1$.

We shall first prove that the theorem is valid for all ideals $I$ in $F[X]$ of the form $\left(I^{\prime}, X_{r}^{k}\right)$ where $I^{\prime}$ is a multihomogeneous, $\left(X_{1}, \ldots, X_{r-1}\right)$-primary ideal in $F\left[X_{1}, \ldots, X_{r-1}\right]$. Note that in this case

$$
L(I)=k L\left(I^{\prime}\right)
$$

for there is a $F$-isomorphism $F\left[X_{1}, \ldots, X_{r-1}\right] / I^{\prime} \rightarrow\left(I^{\prime}, X_{r}^{\nu-1}\right) /\left(I^{\prime}, X_{r}^{\nu}\right) \quad(1 \leqslant \nu \leqslant k)$ induced by multiplication with $X_{r}^{\nu-1}$. By our inductive hypothesis we can apply the theorem to the ring $Q /\left(x_{r}\right)$. In this way we get

$$
L(I)=L\left(\left(I^{\prime}, x_{r}^{k}\right) /\left(x_{r}^{k}\right)\right) \geqslant L\left(I^{\prime}\right) e\left(\left(x_{1}, \ldots, x_{r-1}, x_{r}^{k}\right) /\left(x_{r}^{k}\right)\right) .
$$

By Lemma 1 and the formula (1.1), we have

$$
e\left(\left(x_{1}, \ldots, x_{r-1}, x_{r}^{k}\right) /\left(x_{r}^{k}\right)\right) \geqslant e\left(x_{1}, \ldots, x_{r-1}, x_{r}^{k}\right)=k e\left(x_{1}, \ldots, x_{r}\right) .
$$

Putting together (2.2), (2.3), and (2.1), we obtain

$$
L(I) \geqslant L(I) e\left(x_{1}, \ldots, x_{r}\right)
$$

It remains to show that we have equality here only if $Q$ is a Cohen-Macauley ring. By hypothesis we have equality in (2.2) only if $Q /\left(x_{r}^{k}\right)$ is a Cohen-Macauley ring, hence only if

$$
L\left(x_{1}, \ldots, x_{r-1}, x_{r}^{k}\right)=e\left(\left(x_{1}, \ldots, x_{r-1}, x_{r}^{k}\right) /\left(x_{r}^{k}\right)\right) .
$$




\section{CH. LECH, Note on multiplicities of ideals}

Thus equality holds in (2.2) and (2.3) only if

$$
L\left(x_{1}, \ldots, x_{r-1}, x_{r}^{k}\right)=e\left(x_{1}, \ldots, x_{r-1}, x_{r}^{k}\right),
$$

i.e., only if $Q$ is a Cohen-Macauley ring. This completes the proof for ideals $I$ of the type $\left(I^{\prime}, X_{r}^{k}\right)$.

Now we use induction on the number $k=\min \left\{x \mid X_{r}^{*} \in I\right\}$. If $k=1$, then $I$ has necessarily the form $\left(I^{\prime}, X_{r}\right)$, and we are in the case just treated. Suppose that $k \geqslant 2$ and that the theorem is true when $I$ is replaced by an ideal containing $X_{r}^{k-1}$. Put

$$
I^{\prime}=\left(I: X_{r}^{k-1}\right) \cap F\left[X_{1}, \ldots, X_{r-1}\right]
$$

Then $I^{\prime}$ is a multihomogeneous, $\left(X_{1}, \ldots, X_{r-1}\right)$-primary ideal in $F\left[X_{1}, \ldots, X_{r-1}\right]$. We have

$$
L(I)=L\left(I, X_{r}^{k-1}\right)+L\left(I^{\prime}\right),
$$

for there is a $F$-isomorphism $F\left[X_{1}, \ldots, X_{r-1}\right] / I^{\prime} \rightarrow\left(I, X_{r}^{k-1}\right) / I$ induced by multipilication with $X_{r}^{k-1}$. Taking into account that $I$ and $I^{\prime}$ are multihomogeneous ideals, we further derive that

Hence

$$
I=\left(I, X_{r}^{k-1}\right) \cap\left(I^{\prime}, X_{r}^{k}\right) .
$$

$$
I \subseteq\left(\bar{I}, x_{r}^{k-1}\right) \cap\left(\bar{I}^{\prime}, x_{r}^{k}\right) \text {. }
$$

If we write $\mathfrak{q}_{1} \cap \mathfrak{q}_{2}$ for the right hand side of this inclusion, then, as is seen from the $Q$-isomorphism $\left(\mathfrak{q}_{1}+\mathfrak{q}_{2}\right) / \mathfrak{q}_{2} \rightarrow \mathfrak{q}_{1} / \mathfrak{q}_{1} \cap \mathfrak{q}_{2}$, we have $L\left(\mathfrak{q}_{1} \cap \mathfrak{q}_{2}\right)=L\left(\mathfrak{q}_{1}\right)+$ $+L\left(\mathfrak{q}_{2}\right)-L\left(\mathfrak{q}_{1}+\mathfrak{q}_{2}\right)$. Thus we deduce that

$$
L(\bar{I}) \geqslant L\left(\bar{I}, x_{r}^{k-1}\right)+L\left(\bar{I}^{\prime}, x_{r}^{k}\right)-L\left(\bar{I}^{\prime}, x_{r}^{k-1}\right) .
$$

We shall estimate separately the terms $L\left(I, x_{r}^{k-1}\right)$ and $\bar{L}\left(I^{\prime}, x_{r}^{k}\right)-L\left(\bar{I}^{\prime}, x_{r}^{k-1}\right)$ on the right hand side of this inequality. By the inductive hypothesis,

$$
L\left(\bar{I}, x_{r}^{k-1}\right) \geqslant L\left(I, X_{r}^{k-1}\right) e\left(x_{1}, \ldots, x_{r}\right) .
$$

According to what we have proved for ideals of the type $\left(I^{\prime}, X_{r}^{k}\right)$, we have, for every $n$,

$$
\sum_{p=1}^{n}\left(L\left(I^{\prime}, x_{r}^{p}\right)-L\left(I^{\prime}, x_{r}^{\nu-1}\right)\right)=L\left(I^{\prime}, x_{r}^{n}\right) \geqslant n L\left(I^{\prime}\right) e\left(x_{1}, \ldots, x_{r}\right) .
$$

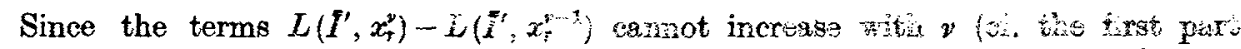
of the proof of Lemma 1), it follows that they all must be larger than or equal to $L\left(I^{\prime}\right) e\left(x_{1}, \ldots, x_{r}\right)$. In particular,

$$
L\left(\tilde{I}^{\prime}, x_{r}^{k}\right)-L\left(I^{\prime}, x_{r}^{k-1}\right) \geqslant L\left(I^{\prime}\right) e\left(x_{1}, \ldots, x_{r}\right) .
$$

Combining (2.5), (2.6), (2.7), and (2.4), we obtain

$$
L(I) \geqslant L(I) e\left(x_{1}, \ldots, x_{r}\right) .
$$


Here equality can hold only if equality holds in (2.6), hence, by the inductive hypothesis, only if $Q$ is a Cohen-Macauley ring. Thus the proof is complete.

Remark. If $I_{1}$ and $I_{2}$ are multihomogeneous, $\left(X_{1}, \ldots, X_{r}\right)$-primary ideals such that $I_{1} \subset I_{2}$ and $L\left(I_{1}\right)-L\left(I_{2}\right)=1$, then it is not necessarily true that $L\left(\bar{I}_{1}\right)-$ $-L\left(I_{2}\right) \geqslant e\left(x_{1}, \ldots, x_{r}\right)$. Example: Let $k$ be a field and $k\left[\left[z_{1}, z_{2}\right]\right]$ a ring of formal power series over $k$. Let $Q$ be the subring of $k\left[\left[z_{1}, z_{2}\right]\right]$ whose elements have the form $\alpha_{00}+\sum_{v+\mu \geqslant 2} \alpha_{v \mu} z_{1}^{\nu} z_{2}^{\mu}\left(\alpha_{\nu \mu} \in k\right)$. Take $x_{1}=z_{1}^{2}, x_{2}=z_{2}^{2}, I_{1}=\left(X_{1}^{2}, X_{2}^{2}\right), I_{2}=$ $=\left(X_{1}^{2}, X_{1} X_{2}, X_{2}^{2}\right)$. Simple direct calculations show that $e\left(x_{1}, x_{2}\right)=4, L\left(\bar{I}_{1}\right)-$ $-\bar{L}\left(\bar{I}_{2}\right)=2$.

Theorem 2. Let $I_{1}, I_{2}, I_{3}, \ldots$ be a sequence of multihomogeneous, $\left(X_{1}, \ldots, X_{r}\right)$ primary ideals in $F[X]$. Suppose that $\lim _{n \rightarrow \infty} L\left(I_{n}\right)=\infty$ and that, for exery fixed $N$,

Thers

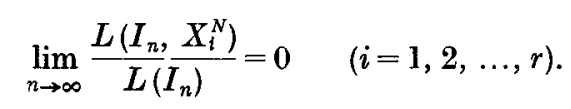

$$
\text { Then } e\left(x_{1}, \ldots, x_{r}\right)=\lim _{n \rightarrow \infty} \frac{L\left(I_{n}\right)}{L\left(I_{n}\right)} \text {. }
$$

By Theorem 1,

$$
\frac{L\left(\bar{I}_{n}\right)}{L\left(I_{n}\right)} \geqslant e\left(x_{1}, \ldots, x_{r}\right) \text {. }
$$

It therefore suffices to show that

$$
\varlimsup_{n \rightarrow \infty} \frac{L\left(\bar{I}_{n}\right)}{L\left(I_{n}\right)} \leqslant e\left(x_{1}, \ldots, x_{r}\right) .
$$

To do this we apply the formula (1.2) with $\mathfrak{q}=\bar{I}_{n}$. (We must then make a strict distinction between the symbols $I_{v}$ and $I_{v}(\quad)$ ) Evidently

$$
L\left(I_{v}\left(\bar{I}_{n}\right)\right) \leqslant L\left(I_{n}\right) \quad(v=0,1, \ldots, l-1) .
$$

By the same lexicographica] argument as in [2], p. 305, one can further show that, for those values of $v$ for which $I_{\nu}(0) \neq(0)$, we have

$$
L\left(I_{v}\left(\bar{I}_{n}\right)\right)=o\left(L\left(I_{n}\right)\right) \quad \text { for } n \rightarrow \infty .
$$

Inserting (2.9) and (2.10) in (1.2) we get (2.8). This completes the proof.

Asollary, Let a and $h$ be analytically disjoint ideals in $Q$, and suppose that $\mathfrak{a}+\mathfrak{b}$ is primary to the maximai idea of $Q$. Then

$$
e(\mathfrak{a}+\mathfrak{b})=\lim _{n, m \rightarrow \infty} \frac{L\left(\mathfrak{a}^{n}+\mathfrak{b}^{n}\right)}{\left(m^{l_{1}} / l_{1} !\right)\left(n^{l_{1}} / l_{2} !\right)},
$$

where $l_{1}$ and $l_{2}$ are the analytic spreads of $\mathfrak{a}$ and $\mathfrak{b}$ respectively 1 .

\footnotetext{
1 I owe to Dr. P. Roquette a suggestion that this result should be true, and this gave the impulse to the results of the present section.
} 
In the special case where $\mathfrak{a}=\left(x_{1}, \ldots, x_{l_{1}}\right), \mathfrak{b}=\left(x_{l_{1}+1}, \ldots, x_{l_{1}+l_{3}}\right)$, the corollary is a direct consequence of the theorem. By using reductions of ideals one can then pass to the general case (cf. [7]).

\section{Ideals of highest power products}

Let $Q$ be a local ring and let $\mathfrak{m}=\left(u_{1}, \ldots, u_{s}\right)$ be its maximal ideal. Put $K=Q / \mathrm{m}$ and form the polynomial ring $K[X]=K\left[X_{1}, \ldots, X_{s}\right]$. With each ideal $a$ in $Q$ we shall associate a multihomogeneous ideal $I(a)$ in $K[X]$. This will be done by first passing from $\mathfrak{a}$ to its form ideal with respect to $\mathfrak{m}=\left(u_{1}, \ldots, u_{s}\right)$ and then applying a transformation (denoted by $\psi$ below) which transforms every homogeneous ideal of $K[X]$ into a multihomogeneous ideal.

To begin, let us recall the notion of form ideal. Let $\mathfrak{a}$ be an ideal of $Q$. If $\phi(X)$ is an element of the polynomial ring $Q[X]=Q\left[X_{1}, \ldots, X_{s}\right]$, let $\bar{\phi}(X)$ denote its image under the natural map $Q[X] \rightarrow K[X]$, and let $\phi(u)$ be the element in $Q$ obtained from $\phi(X)$ by substituting $u_{1}, \ldots, u_{s}$ for $X_{1}, \ldots, X_{s}$. The set

$$
\{\bar{\phi}(X) \mid \phi(X) \text { form of } Q[X], \phi(u) \in \mathfrak{a}\}
$$

is the set of forms in a homogeneous ideal of $K[X]$. This ideal is called the form ideal of $\mathfrak{a}$ with respect to $\mathfrak{m}=\left(u_{1}, \ldots, u_{s}\right)$ and will be denoted by $\overline{\mathfrak{a}}$. Let $\mathfrak{b}$ be another ideal of $Q$. It follows directly from the definitions of $\overline{\mathfrak{a}}$ and $\overline{\mathfrak{b}}$ that

$$
\mathfrak{a} \subseteq \mathfrak{b} \text { implies } \overline{\mathfrak{a}} \subseteq \overline{\mathfrak{b}}
$$

and that

$$
\overline{\mathfrak{a}} \overline{\mathfrak{b}} \subseteq \overline{\mathfrak{a} \mathfrak{b}} \text {. }
$$

We shall derive one further property of the mapping $\mathfrak{a} \rightarrow \overline{\mathfrak{a}}$. Denote by $\boldsymbol{F}_{\mu}$ the $K$-module consisting of the forms of degree $\mu$ in $K[X]$. There is a $K$-homomorphism of $F_{\mu}$ onto the canonical $K$-module $\left(\mathfrak{m}^{\mu}+\mathfrak{a}\right) /\left(\mathfrak{m}^{\mu+1}+\mathfrak{a}\right)$ which maps $X_{1}^{\sigma_{1}} \ldots X_{s}^{\sigma_{s}}\left(\sigma_{1}+\cdots+\sigma_{s}=\mu\right)$ on the residue class represented by $u_{1}^{\sigma_{1}} \ldots u_{s}^{\sigma_{s}}$. It is readily verified that the kernel of this homomorphism is $F_{\mu} \cap \overline{\mathfrak{a}}$. Thus

$$
\operatorname{dim}_{K}\left(F_{\mu} / F_{\mu} \cap \overline{\mathfrak{a}}\right)=\operatorname{dim}_{K}\left(\left(\mathrm{~m}^{\mu}+\mathfrak{a}\right) /\left(\mathfrak{n t}^{\mu+1}+\mathfrak{a}\right)\right) .
$$

Next we introduce a preliminary transformation $\varphi$. Extend the field $K$ by adjunction of a set $\left\{a_{i k}\right\}_{i, k=1,2, \ldots . .}$ of independent indeterminates over $K$. Call the extension $L$. Let $\varphi$ be the automorphism of $L[X]$ over $L$ (i.e. leaving the elements of $L$ fixed) defined by.

$$
X_{i} \rightarrow \sum_{k=1}^{s} a_{i k} X_{k} \quad(i=1,2, \ldots, s)
$$

Now let $\mathfrak{A}$ be a homogeneous ideal of $K[X]$. Order the power products of $X_{1}, \ldots, X_{s}$ lexicographically by prescribing that $X_{1}^{\sigma_{1}} \ldots X_{s}^{\sigma_{s}}$ is higher than $X_{1}^{\tau_{1}} \ldots X_{s}^{\tau_{s}}$ if the last of the differences

$$
\sigma_{1}-\tau_{1}, \sigma_{2}-\tau_{2}, \ldots, \sigma_{s}-\tau_{s}
$$


which is different from zero, is positive. Associate with each form in the ideal $\varphi(\mathfrak{A} L[X])$ its highest power product. The set of power products which arise in this way is the set of power products in a multihomogeneous ideal of $K[X]$. We denote this ideal by $\psi(\mathfrak{A})$. The mapping $\mathfrak{A} \rightarrow \psi(\mathfrak{A})$ can then be split up as follows :

$$
\mathfrak{A} \rightarrow \mathfrak{A} L[X] \rightarrow \varphi(\mathfrak{A} L[X]) \rightarrow \psi(\mathfrak{A}) .
$$

By considering these mappings separately one can readily verify the following three statements, where $\mathscr{A}$ and $\mathfrak{B}$ denote homogeneous ideals of $K[X]$ :

$$
\begin{gathered}
\mathfrak{A} \subseteq \mathfrak{B} \text { implies } \psi(\mathfrak{A}) \subseteq \psi(\mathfrak{B}) \\
\psi(\mathfrak{U}) \psi(\mathfrak{B}) \subseteq \psi(\mathfrak{U} \mathfrak{B}) \\
\operatorname{dim}_{K}\left(F_{\mu} / F_{\mu} \cap \psi(\mathfrak{A})\right)=\operatorname{dim}_{K}\left(F_{\mu} / F_{\mu} \cap \mathfrak{U}\right)
\end{gathered}
$$

Because of the preliminary transformation $\varphi$, the multihomogeneous ideals in $K[X]$ which can be written on the form $\psi(\mathfrak{A})$ will have some special properties. We record two of these in a lemma.

Lemma 2. Every ideal of the form $\psi(\mathfrak{A})$, where $\mathfrak{A}$ denotes an arbitrary homogeneous ideal of $K[X]$, has the properties $(A)$ and $(B)$ below.

(A) Assume $\psi(\mathfrak{A}) \neq(0)$. Let $k$ be the least integer such that

$$
\psi(\mathfrak{A}) \cap K\left[X_{1}, \ldots, X_{k}\right] \neq(0) .
$$

Then, for some natural number $n$,

$$
\left(X_{k}, \ldots, X_{s}\right)^{n} \subseteq \psi(\mathfrak{A}) \subseteq\left(X_{k}, \ldots, X_{s}\right)
$$

In particular, $\left(X_{k}, \ldots, X_{s}\right)$ is the only minimal prime ideal of $\psi(\mathfrak{A})$. (For $k=0$ the ideal $\left(X_{k}, \ldots, X_{s}\right)$ should be interpreted as (1).)

(B) If

then also

$$
X_{1}^{\sigma_{1}} \ldots X_{s}^{\sigma_{s}} \in \psi(\mathfrak{A}),
$$

$$
X_{m}^{\left(\sigma_{1}+\cdots+\sigma_{m}\right)} X_{m+1}^{\sigma_{m+1}} \ldots X_{s}^{\sigma_{s}} \in \psi(\mathfrak{A}) \quad(1 \leqslant m \leqslant s)
$$

The proof of the two properties will be based on one and the same principle, which in outline runs as follows. Suppose that $f$ is a form of $\varphi(\mathfrak{A} L[X])$, and let $\varphi^{\prime}$ be a transformation of the same type as $\varphi$. Then, if $\varphi$ and $\varphi^{\prime}$ are in a natural sense independent of each other, the highest power product of $\varphi^{\prime}(f)$ will belong to $\psi(\mathfrak{A})$.

We now set forth the details of this principle. Let $\left(b_{i k}\right)$ be a non-singular $s \times s$-matrix with elements in an extension field $M$ of $L$. Let $\left(c_{i k}\right)$ be the product matrix $\left(a_{i k}\right)\left(b_{i k}\right)$. With a slight change in the definition of $\varphi$, denote by $\varphi, \varphi^{\prime}$, and $\varphi^{\prime \prime}$ the automorphisms of $M[X]$ over $M$ defined by

$$
X_{i} \rightarrow \sum_{k=1}^{s} a_{i k} X_{k}, \quad X_{i} \rightarrow \sum_{k=1}^{s} b_{i k} X_{k}, \quad \text { and } \quad X_{i} \rightarrow \sum_{k=1}^{s} c_{i k} X_{k}
$$


respectively. Then $\varphi^{\prime \prime}$ is equal to the composition $\varphi^{\prime} \circ \varphi$. Denote by $L^{\prime}$ and $L^{\prime \prime}$ the extensions of $K$ generated by the $b_{i k}$ and the $c_{i k}$ respectively.

Assume that $L$ and $L^{\prime}$ are free over $K$, i.e. that the $a_{i k}$ are independent indeterminates over $L^{\prime}$. (This is the condition on $\varphi$ and $\varphi^{\prime}$ which we have alluded to above.) Then the $c_{i k}$ are independent indeterminates over $K$. For, since the $a_{i k}$ are independent indeterminates over $L^{\prime}$, there is an endomorphism (specialization) of $L^{\prime}\left[a_{11}, a_{12}, \ldots, a_{s s}\right]$ over $L^{\prime}$ which maps $\left(a_{i k}\right)$ on $\left(a_{i k}\right)\left(b_{i k}\right)^{-1}$. (Strictly speaking, the elements of the first matrix are mapped on the corresponding elements of the second one.) This endomorphism then maps $\left(c_{i k}\right)=$ $=\left(a_{i k}\right)\left(b_{i k}\right)$ on $\left(a_{i k}\right)$. It follows that the $c_{i k}$ are independent indeterminates over $L^{\prime}$, hence, a fortiori, over $K$. As a consequence, there is an isomorphism of $L^{\prime \prime}$ onto $L$ over $K$ which maps $\left(c_{i k}\right)$ on $\left(a_{i k}\right)$, hence takes the $L^{\prime \prime}[X]$-restriction of $\varphi^{\prime \prime}$ into the $L[X]$-restriction of $\varphi$.

Let now $f$ be a form of $\varphi(\mathfrak{A} L[X])$, and let $X^{\langle 0\rangle}$ be the highest power product of $\varphi^{\prime}(f)$. We have $\varphi^{\prime}(f) \in \varphi^{\prime} \circ \varphi(\mathfrak{A} L[X])=\varphi^{\prime \prime}(\mathfrak{U} L[X])$. Thus $X^{(0)}$ is the highest power product of a form belonging to $\varphi^{\prime \prime}(\mathfrak{A} M[X])=\varphi^{\prime \prime}\left(\mathfrak{A} L^{\prime \prime}[X]\right) \cdot M[X]$. It follows that $X^{(0)}$ is the highest power product of a form in $\varphi^{\prime \prime}\left(\mathfrak{A} L^{\prime \prime}[X]\right)$, hence, in view of the isomorphism of $L^{\prime \prime}$ onto $L$, of a form in $\varphi(\mathfrak{A} L[X])$. Thus $X^{(0)} \in \psi(\mathfrak{A})$. This establishes our principle.

It remains to prove (A) and (B) by making suitable choices of $f$ and $\varphi^{\prime}$.

Proof of (A). The case $k=0$ is trivial. Assume $k>0$, and let $l$ be an integer satisfying $k \leqslant l \leqslant s$. Since $\psi(\mathfrak{A}) \cap K\left[X_{1}, \ldots, X_{k}\right] \neq(0)$, we have

$$
\varphi(\mathfrak{A} L[X]) \cap L\left[X_{1}, \ldots, X_{k}\right] \neq(0) .
$$

Take $f$ as a non-zero form in $\varphi(\mathfrak{A} L[X]) \cap L\left[X_{1}, \ldots X_{k}\right]$, say of degree $d$. As $t \notin L$, we have $d>0$. Define $\varphi^{\prime}$ by

$$
X_{\imath} \rightarrow X_{i}+b_{i} X_{l} \quad(i=1,2, \ldots, s),
$$

where $b_{1}, \ldots, b_{s}$ are independent indeterminates over $L$. Then $X_{l}^{d}$ is the highest power product of $\varphi^{\prime}(f)$. By varying $l$ we see that $\left(X_{k}^{d}, \ldots, X_{s}^{d}\right)$ is contained in $\psi(\mathfrak{A})$, hence also $\left(X_{k}, \ldots, X_{s}\right)^{d s}$. This proves the left inclusion. The right inclusion is an immediate consequence of the definition of $k$ and the fact that $\psi(\mathfrak{A})$ is multihomogeneous.

Proof of (B). Choose $f$ such that $X_{1}^{o_{1}} \ldots X_{s}^{o_{2}}$ is the highest power product of $f$. Define $\varphi^{\prime}$ by

$$
\begin{array}{ll}
X_{i} \rightarrow X_{i}+c_{i} X_{m} & (1 \leqslant i \leqslant m), \\
X_{i} \rightarrow X_{i} & (m<i \leqslant s),
\end{array}
$$

where $c_{1}, \ldots, c_{m}$ are independent indeterminates over $L$. Then $X_{m}^{\sigma_{1}+\cdots+\sigma_{m}} X_{m+1}^{\sigma_{m+1}} \ldots X_{s}^{\sigma_{s}}$ is the highest power product of $\varphi^{\prime}(f)$, which gives the result.

The proof of Lemma 2 is complete. 
We now compose the mappings $\mathfrak{a} \rightarrow \overline{\mathfrak{a}}$ and $\mathfrak{A} \rightarrow \psi(\mathfrak{A}):$ if $\mathfrak{a}$ is an ideal of the local ring $Q$, we define $I(\mathfrak{a})=\psi(\overline{\mathfrak{a}})$. The ideal $I(\mathfrak{a})$ is then a multihomogeneous ideal of $K[X]$ which has the properties (A) and (B) of Lemma 2. Further, combining (3.1), (3.2), and (3.3) with (3.4), (3.5), and (3.6), we see that

$$
\begin{gathered}
\mathfrak{a} \subseteq \mathfrak{b} \text { implies } I(\mathfrak{a}) \subseteq I(\mathfrak{b}) \\
I(\mathfrak{a}) I(\mathfrak{b}) \subseteq I(\mathfrak{a} \mathfrak{b}) \\
\operatorname{dim}_{K}\left(F_{\mu} / F_{\mu} \cap I(\mathfrak{a})\right)=\operatorname{dim}_{K}\left(\left(\mathfrak{m}^{\mu}+\mathfrak{a}\right) /\left(\mathrm{m}^{\mu+1}+\mathfrak{a}\right)\right) .
\end{gathered}
$$

We shall derive some consequences of the formula (3.9), which expresses a length-preserving property of the mapping $\mathfrak{a} \rightarrow I(\mathfrak{a})$. Denote by $\mathfrak{M}$ the ideal $\left(X_{1}, \ldots, X_{s}\right)$ in $K[X]$. From the canonical $K$-homomorphism $\mathfrak{M}^{\mu} / \mathfrak{M}^{\mu+1} \rightarrow F_{\mu}$ we get the induced isomorphism

$$
\left(\mathfrak{M}^{\mu}+I(\mathfrak{a})\right) /\left(\mathfrak{M}^{\mu+1}+I(\mathfrak{a})\right) \simeq \mathfrak{M}^{\mu} /\left(\left(\mathfrak{M}^{\mu} \cap I(\mathfrak{a})\right)+\mathfrak{M}^{\mu+1}\right) \simeq F_{\mu} / F_{\mu} \cap I(\mathfrak{a}) .
$$

Combining this isomorphism with (3.9), we get

$$
\operatorname{dim}_{K}\left(\left(\mathfrak{M}^{\mu}+I(\mathfrak{a})\right) /\left(\mathfrak{M}^{\mu+1}+I(\mathfrak{a})\right)\right)=\operatorname{dim}_{K}\left(\left(\mathfrak{m}^{\mu}+\mathfrak{a}\right) /\left(\mathfrak{m}^{\mu+1}+\mathfrak{a}\right)\right) .
$$

Thus, summing from $\mu=0$ to $\mu=n-1$,

This means that

$$
L\left(\mathfrak{M}^{n}+I(\mathfrak{a})\right)=L\left(\mathfrak{m}^{n}+\mathfrak{a}\right) .
$$

$$
\begin{aligned}
\operatorname{dim} \mathfrak{a} & =\operatorname{dim}\left(I(\mathfrak{a}) K[X]_{\mathfrak{M})},\right. \\
e(\mathfrak{m} / \mathfrak{a}) & =e(\mathfrak{M} / I(\mathfrak{a}))
\end{aligned}
$$

(cf. the definition of multiplicity).

The right hand sides of (3.11) and (3.12) can be expressed in terms of the special structure of $I(\mathfrak{a})$. Let $\left(X_{d^{\prime}+1}, \ldots, X_{s}\right)$ be the minimal prime ideal of $I(\mathfrak{a})$ (cf. Lemma 2). Then

$$
\operatorname{dim}\left(I(\mathfrak{a}) K[X]_{\mathfrak{M}}\right)=\operatorname{dim}\left(\left(X_{d^{\prime}+1}, \ldots, X_{s}\right) K[X]_{\mathfrak{M}}\right)=d^{\prime},
$$

and, by the additivity formula,

$$
\begin{aligned}
e(\mathfrak{M} / I(\mathfrak{a}))=e\left(\mathfrak{M} /\left(X_{d^{\prime}+1}, \ldots, X_{s}\right)\right) \cdot L\left(I(\mathfrak{a}) ;\left(X_{d^{\prime}+1}, \ldots, X_{s}\right)\right)= \\
\quad=L\left(I(\mathfrak{a}) ;\left(X_{d^{\prime}+1}, \ldots, X_{s}\right) .\right.
\end{aligned}
$$

In particular, if $\left(X_{d+1}, \ldots, X_{s}\right)$ is the minimal prime ideal of $I(0)$, then by (3.11)-(3.14),

$$
\begin{aligned}
\operatorname{dim} Q & =d \\
e(Q) & =L\left(I(0) ;\left(X_{d+1}, \ldots, X_{s}\right)\right)
\end{aligned}
$$


CH. LECH, Note on multiplicities of ideals

In (3.14) the ideal $\mathfrak{M}$ can be replaced by $\left(X_{c+1}, \ldots, X_{s}\right)\left(0 \leqslant c \leqslant d^{\prime}\right)$. Thus, for $\mathfrak{a}=(0)$,

$$
e\left(\left(X_{c+1}, \ldots, X_{d}\right) / I(0)\right)=e(Q) \quad(0 \leqslant c \leqslant d) .
$$

Finally, if $q$ is a m-primary ideal, we get from (3.10), by taking $n$ large,

$$
L(I(\mathfrak{q}))=L(\mathfrak{q})
$$

\section{Applications of the preceding section}

Theorem 3. Let $q$ be a primary ideal in a local ring $Q$. Assume that $\operatorname{dim} \mathfrak{q}+\operatorname{rank} q=\operatorname{dim} Q$. Put $\operatorname{rank} \mathfrak{q}=m$. Then

$$
\frac{e(\mathfrak{q})}{L(\mathfrak{q})} \leqslant(m !) e(Q)
$$

Proof. 1) Let $F[X]=F\left[X_{1}, \ldots, X_{d}\right]$ be a polynomial ring over a field $F$, and let $\mathfrak{A}$ be a multihomogeneous, $\left(X_{1}, \ldots, X_{d}\right)$-primary ideal of $F[X]$. Denote by $\mathfrak{A}_{n}$ the ideal in $F[X]$ generated by the $n^{\text {th }}$ powers of the power products contained in $\mathfrak{A}$. Then $L\left(\mathfrak{A}_{n}\right)=n^{d} L(\mathfrak{A})$. For, if $\mathfrak{A}^{\prime}$ is a multihomogeneous ideal containing $\mathfrak{A}$ such that $L\left(\mathfrak{U}^{\prime}\right)=L(\mathfrak{U})-1$, and if $\mathfrak{A}_{n}^{\prime}$ is defined in analogy with $\mathfrak{A}_{n}$, then $\mathfrak{A}_{n}^{\prime} / \mathfrak{A}_{n}$ is $F[X]$-isomorphic to $F[X] /\left(X_{1}^{n}, \ldots, X_{d}^{n}\right)$. This gives $L\left(\mathfrak{A}_{n}\right)=$ $=L\left(\mathfrak{A}_{n}^{\prime}\right)+n^{d}$, and thus, by induction, $L\left(\mathfrak{A}_{n}\right)=n^{d} L(\mathfrak{A})$. Since $\mathfrak{A}_{n} \subseteq \mathfrak{A}^{n}$, we get $L\left(\mathfrak{A}^{n}\right) \leqslant n^{d} L(\mathfrak{A})$. Hence

$$
\frac{e(\mathfrak{A})}{L(\mathfrak{A})}=\lim _{n \rightarrow \infty} \frac{(d !) L\left(\mathfrak{A}^{n}\right)}{n^{d} L(\mathfrak{U})} \leqslant d ! .
$$

Let us remark that, for $d>1$, we actually have strict inequality in (4.1).

2) Assume that $\operatorname{dim} \mathfrak{q}=0$. Put $\operatorname{dim} Q=d$. We shall apply the mapping $\mathfrak{a} \rightarrow I(\mathfrak{a})$ of Section 3 (with respect to an arbitrary basis of the maximal ideal of $Q$ ). By (3.18), (3.7), and (3.8),

$$
L\left(q^{n}\right)=L\left(I\left(q^{n}\right)\right) \leqslant L\left(I(q)^{n}+I(0)\right) .
$$

Hence, applying the additivity formula and the formulae (3.15) and (3.16),

$$
\begin{aligned}
e(\mathfrak{q})=\lim _{n \rightarrow \infty} & \frac{L\left(\mathfrak{q}^{n}\right)}{n^{d} / d !} \leqslant \lim _{n \rightarrow \infty} \frac{L\left(I(\mathfrak{q})^{n}+I(0)\right)}{n^{d} / d !}= \\
& =e(I(\mathfrak{q}) / I(0))=e\left(I(\mathfrak{q})+\left(X_{d+1}, \ldots, X_{s}\right) /\left(X_{d+1}, \ldots, X_{s}\right)\right) \cdot e(Q) .
\end{aligned}
$$

Put

$$
\overline{I(q)}=\left(I(q)+\left(X_{d+1}, \ldots, X_{s}\right)\right) /\left(X_{d+1}, \ldots, X_{s}\right) .
$$

In view of the canonical isomorphism $K[X] /\left(X_{d+1}, \ldots, X_{s}\right) \rightarrow K\left[X_{1}, \ldots, X_{d}\right]$, we can regard $\overline{I(q)}$ as a multihomogeneous, $\left(X_{1}, \ldots, X_{d}\right)$-primary ideal of $K\left[X_{1}, \ldots, X_{d}\right]$. Thus, by (4.1),

$$
\frac{e(\overline{I(\mathfrak{q})})}{L(\overline{I(\mathfrak{q}))}} \leqslant d !
$$


Combining (4.2), (4.3), and (4.4) with the obvious fact that

we obtain

$$
L(\overline{I(q)}) \leqslant L(I(q))=L(\mathfrak{q}),
$$

$$
\frac{e(\mathfrak{q})}{L(\mathfrak{q})} \leqslant(d !) e(Q)
$$

3) Assume $\operatorname{dim} \mathfrak{q}=d^{\prime}>0$. Again we shall apply the mapping $\mathfrak{a} \rightarrow I(\mathfrak{a})$ of Section 3. Observe that, because $\operatorname{dim} q+\operatorname{rank} q=\operatorname{dim} Q$, we have $\operatorname{rank}(I(q) / I(0))=$ $=\operatorname{rank} \mathfrak{q}=m$ (cf. (3.11) and (3.13)).

Let $\mathfrak{p}$ be the prime ideal belonging to $q$. By the additivity formula we have

Hence

$$
e\left(\mathrm{~m} / \mathfrak{q}^{n}\right)=e(\mathrm{~m} / \mathfrak{p}) L\left(\mathfrak{q}^{n} ; \mathfrak{p}\right) \quad(n=1,2,3, \ldots) .
$$

$$
\frac{L\left(\mathfrak{q}^{n} ; \mathfrak{p}\right)}{L(\mathfrak{q} ; \mathfrak{p})}=\frac{e\left(\mathfrak{m} / \mathfrak{q}^{n}\right)}{e(\mathfrak{m} / \mathfrak{q})}
$$

Similarly, in the ring $K[X]$, with $\mathfrak{M}=\left(X_{1}, \ldots, X_{s}\right)$,

$$
\frac{L\left(I(\mathfrak{q})^{n}+I(0) ;\left(X_{d^{\prime}+1}, \ldots, X_{s}\right)\right)}{\left.L\left(I(\mathfrak{q}) ; X_{d^{\prime}+1}, \ldots, X_{s}\right)\right)}=\frac{e\left(\mathfrak{M} /\left(I(\mathfrak{q})^{n}+I(0)\right)\right)}{e(\mathfrak{M} / I(\mathfrak{q}))}
$$

Comparing the right hand sides of (4.6) and (4.7), and using (3.12), we see that the expression (4.6) is less than or equal to the expression (4.7). Thus, multiplying (4.6) and (4.7) by $(m !) / n^{m}$, and passing to the limit, we get

$$
\frac{e(\mathfrak{q})}{L(\mathfrak{q})} \leqslant \frac{e\left(I(\mathfrak{q}) / I(0) ;\left(X_{d^{\prime}+1}, \ldots, X_{s}\right) / I(0)\right)}{L\left(I(\mathfrak{q}) / I(0) ;\left(X_{d^{\prime}+1}, \ldots, X_{s}\right) / I(0)\right)} .
$$

Applying (4.5) to the right hand side of this inequality, we obtain, by (3.17),

$$
\frac{e(\mathfrak{q})}{L(\mathfrak{q})} \leqslant(m !) e(Q)
$$

This completes the proof of Theorem 3 .

Corollary. If $\left(Q_{0}, Q\right)$ is a flat couple of d-dimensional local rings, then

$$
e\left(Q_{0}\right) \leqslant d ! e(Q) .
$$

Proof. Apply Theorem 3 with $q=\mathfrak{m}_{0} Q$ where $\mathfrak{m}_{0}$ is the maximal jdeal of $Q_{0}$, and observe that $e\left(\mathfrak{m}_{0} Q\right)=e\left(Q_{0}\right) L\left(\mathfrak{m}_{0} Q\right)$ (cf. the appendix).

Theorem 4. If $\left(Q_{0}, Q\right)$ is a flat couple of two-dimensional local rings, then $e\left(Q_{0}\right) \leqslant e(Q)$.

Proof. Let $m_{0}$ and $m$ be the maximal ideals of $Q_{0}$ and $Q$ respectively. Put $K_{0}=Q_{0} / \mathfrak{m}_{0}, K=Q / \mathfrak{m}$. We shall apply the mapping $\mathfrak{a} \rightarrow I(\mathfrak{a})$ of Section 3 to the present ring $Q$. Since $\operatorname{dim} Q=2$, it follows from $(3.15)$ that $\left(X_{3}, \ldots, X_{s}\right)$ is the 
minimal prime ideal of $I(0)$. Let us define $I^{*}(0)$ as the $\left(X_{3}, \ldots, X_{s}\right)$-primary component of $I(0)$. Then, by $(3.16)$,

$$
e(Q)=L\left(I^{*}(0)\right)
$$

Further, $I^{*}(0)$ can be generated by power products of $X_{3}, \ldots, X_{s}$, and $L\left(I^{*}(0)\right)$ is equal to the number of such power products which are not contained in $I^{*}(0)$ (ef. Section 1, (i), and e.g. [5], 4.11).

Define $b$ as the least integer for which

$$
X_{2}^{b} \in I\left(\mathrm{~m}_{0} Q\right)
$$

It then follows from (B) of Lemma 2 that there are no power products of the form $X_{1}^{\nu} X_{2}^{b-1-y}(0 \leqslant \nu \leqslant b-1)$ in $I\left(\mathfrak{m}_{0} Q\right)$. Thus, putting

$$
\mathfrak{q}_{1}=\mathrm{m}_{0} Q+\mathfrak{m}^{b-1}, \quad \mathfrak{q}_{2}=\mathfrak{m}_{0} Q+\mathfrak{m}^{b}
$$

we get from (3.9),

$$
\operatorname{dim}_{K}\left(\mathfrak{q}_{1} / \mathfrak{q}_{2}\right) \geqslant b
$$

(In view of this inequality the number $b$ may be looked upon as a lower estimate of some kind of "breadth" of the ideal $\mathrm{m}_{0} Q$.)

We shall estimate in two ways the number

$$
\operatorname{dim}_{K}\left(\mathfrak{m}_{0}^{n} \mathfrak{q}_{1} / \mathfrak{m}_{0}^{n} \mathfrak{q}_{2}\right)
$$

for large values of $n$. (The $\mathfrak{m}_{0}^{n} \mathfrak{q}_{i}(i=1,2)$ denote ideals of $Q$.)

By (3.18), we have

$$
\operatorname{dim}_{K}\left(\mathfrak{m}_{0}^{n} \mathfrak{q}_{1} / \mathfrak{m}_{0}^{n} \mathfrak{q}_{2}\right)=\operatorname{dim}_{K}\left(I\left(\mathfrak{m}_{0}^{n} \mathfrak{q}_{1}\right) / I\left(\mathfrak{m}_{0}^{n} \mathfrak{q}_{2}\right)\right)
$$

and we may compute the right hand side of this equality, that is, count the number of power products $X_{1}^{\sigma_{1}} \ldots X_{s}^{\sigma_{s}}$ which belong to $I\left(\mathfrak{m}_{0}^{n} \mathfrak{q}_{1}\right)$ but not to $I\left(\mathfrak{m}_{0}^{n} \mathfrak{q}_{2}\right)$. Since $\mathfrak{m}\left(\mathfrak{m}_{0}^{n} \mathfrak{q}_{1}\right) \subseteq \mathfrak{m}_{0}^{n} \mathfrak{q}_{2}$, we have, by (3.8),

i.e.

$$
\begin{gathered}
I(\mathfrak{m}) I\left(\mathfrak{m}_{0}^{n} \mathfrak{q}_{1}\right) \subseteq I\left(\mathfrak{m}_{0}^{n} \mathfrak{q}_{2}\right), \\
\left(X_{1}, \ldots, X_{s}\right) I\left(\mathfrak{m}_{0}^{n} \mathfrak{q}_{1}\right) \subseteq I\left(\mathfrak{m}_{0}^{n} \mathfrak{q}_{2}\right) .
\end{gathered}
$$

This implies that there are no two power products belonging to $I\left(\mathrm{~m}_{0}^{n} \mathfrak{q}_{1}\right)$ but not to $I\left(\mathfrak{m}_{0}^{n} \mathfrak{q}_{2}\right)$ which differ in merely one of the exponents. These power products are then characterized by their associated $(s-1)$-tuples $\left(\sigma_{2}, \ldots, \sigma_{s}\right)$. Thus, to get an upper estimate of $\operatorname{dim}_{K}\left(m_{0}^{n} q_{1} / m_{0}^{n} \mathfrak{q}_{2}\right)$, we may compute the number of elements in the set $S$ of $(s-1)$-tuples $\left(\sigma_{2}, \ldots, \sigma_{s}\right)$ which have the property that, for some $\sigma_{1}, X_{1}^{\sigma_{1}} \ldots X_{s}^{\sigma_{2}}$ does not belong to $I\left(\mathfrak{m}_{0}^{n+1} Q\right) \subseteq I\left(\mathfrak{m}_{0}^{n} \mathfrak{q}_{2}\right)$.

In doing so we shall distinguish between three possibilities for $\left(\sigma_{3}, \ldots, \sigma_{s}\right)$.

(i) $X_{3}^{\sigma_{3}} \ldots X_{s}^{\sigma_{u}} \in I(0)$. Since $I(0) \subseteq I\left(\mathrm{~m}_{0}^{n+1} Q\right)$, it follows that $\left(\sigma_{2}, \ldots, \sigma_{s}\right)$ does not belong to $S$ for any $\sigma_{2}$. 
(ii) $X_{3}^{\sigma_{s}} \ldots X_{s}^{\sigma_{s}} \in I^{*}(0), \quad X_{3}^{\sigma_{3}} \ldots X_{s}^{\sigma_{s}} \notin I(0)$. By (A) of Lemma 2 there are only finitely many $\left(\sigma_{3}, \ldots, \sigma_{s}\right)$ of this kind. For each of these there is some pair $(\alpha, \beta)$ such that $X_{1}^{\alpha} X_{2}^{\beta} X_{3}^{\sigma_{3}} \ldots X_{s}^{\sigma_{s}} \in I(0)$. Then, by (B) of Lemma $2, X_{2}^{\alpha+\beta} X_{3}^{\sigma_{3}} \ldots$ $\ldots X_{s}^{\sigma_{s}} \in I(0)$, so that if $\left(\sigma_{2}, \ldots, \sigma_{s}\right) \in S$, then $\sigma_{2}<\alpha+\beta$.

(iii) $X_{3}^{\sigma_{3}} \ldots X_{s}^{\sigma_{s}} \notin I^{*}(0)$. By $(4.8)$, this case arises for $e(Q)$ different values of $\left(\sigma_{3}, \ldots, \sigma_{s}\right)$. Since, by virtue of $(4.9)$ and $(3.8), X_{2}^{b(n+1)} \in I\left(\mathrm{~m}_{0}^{n+1} Q\right)$, there are, for each such value of $\left(\sigma_{3}, \ldots, \sigma_{s}\right)$, at most $b(n+1)(s-1)$-tuples $\left(\sigma_{2}, \ldots, \sigma_{s}\right)$ in $S$.

Putting together, we get

$$
\operatorname{dim}_{K}\left(m_{0}^{n} q_{1} / m_{0}^{n} q_{2}\right) \leqslant e(Q) b n+O(1) .
$$

On the other hand, since $\left(Q_{0}, Q\right)$ is a flat couple, the module $M=\mathfrak{m}_{0}^{n} Q / \mathfrak{m}_{0}^{n+1} Q$ is a free $\left(Q / \mathrm{m}_{0} Q\right)$-module with a basis of $\operatorname{dim}_{z_{0}}\left(\mathrm{~m}_{0}^{n} / \mathrm{m}_{0}^{n+1}\right)$ elements (cf. the appendix). Hence

$$
\operatorname{dim}_{K}\left(\mathfrak{m}_{0}^{n} \mathfrak{q}_{\mathbf{l}} / \mathfrak{m}_{0}^{n} \mathfrak{q}_{2}\right)=\operatorname{dim}_{K}\left(\mathfrak{q}_{1} M / \mathfrak{q}_{2} M\right)=\operatorname{dim}_{K_{\mathfrak{\imath}}}\left(\mathrm{m}_{0}^{n} / \mathfrak{m}_{0}^{n+1}\right) \cdot \operatorname{dim}_{K}\left(\mathfrak{q}_{1} / \mathfrak{q}_{2}\right) .
$$

Since $\operatorname{dim} Q_{0}=2$,

$$
\operatorname{dim}_{E_{\mathrm{v}}}\left(m_{0}^{n} / \mathrm{m}_{0}^{n+1}\right)=e\left(Q_{0}\right) n+O(1) .
$$

Thus, on account of $(4.10)$,

$$
\operatorname{dim}_{K}\left(\mathfrak{m}_{0}^{n} \mathfrak{q}_{1} / m_{0}^{n} \mathfrak{q}_{2}\right) \geqslant e\left(Q_{0}\right) b n+O(1) .
$$

A comparison of (4.11) and (4.12) gives

$$
e\left(Q_{0}\right) \leqslant e(Q)
$$

which was to be proved.

\section{Flat couples $\left(Q_{0}, Q\right)$ with special conditions on $Q / \mathrm{n}_{0} Q$}

Let $Q$ be a local ring, $\mathfrak{m}$ its maximal ideal, and $q$ a m-primary ideal. Suppose that there is a ring isomarphism

$$
Q / \mathfrak{q} \rightarrow K\left[X_{1}, \ldots, X_{s}\right] /\left(f_{1}, \ldots, f_{s}\right),
$$

where $K$ is a field, $K\left[X_{1} \ldots, X_{s}\right]$ a polynomial ring over $K$, and $\left(f_{1}, \ldots, f_{s}\right) a$ $\left(X_{1}, \ldots, X_{s}\right)$-primary ideal in $K\left[X_{1}, \ldots, X_{s}\right]$. In addition, suppose that $Q$ is equicharacteristic. Then

$$
e(\mathfrak{q}) \leqslant L(\mathfrak{q}) e(\mathrm{~m})
$$

We shall outline a proof of this statement. For $s=0$ we have $\mathfrak{q}=\mathfrak{m}$, and the assertion is trivially true. Thus we can assume that $s>0$. We shall subject the triple $\{Q, \mathfrak{m}, \mathfrak{q}\}$ to a number of successive changes, which will not. affect the values of $e(\mathfrak{m}), L(\mathfrak{q})$, and $e(\mathfrak{q})$, nor the assumptions on $Q / \mathfrak{q}$. Before we list these changes it will be convenient to introduce the homomorphism 
CH. LECH, Note.on multiplicities of ideals

$$
\theta: Q \rightarrow K\left[X_{1}, \ldots, X_{s}\right] /\left(f_{1}, \ldots, f_{s}\right)
$$

defined as the composition of the natural homomorphism $Q \rightarrow Q / \mathfrak{q}$ and the isomorphism (5.1).

(i) Replace $Q$ by $Q^{\prime}=Q\left[z_{1}, \ldots, z_{s}\right]_{\left(\mathfrak{m}, z_{1}, \ldots, z_{s}\right)}$ where $z_{1}, \ldots, z_{s}$ are indeterminates over $Q$. Replace $\mathrm{m}$ by $\mathrm{m}^{\prime}=\left(\mathrm{nt}, z_{1}, \ldots, z_{s}\right) Q^{\prime}$ and $\mathfrak{q}$ by $\mathrm{q}^{\prime}=\left(\mathfrak{q}, z_{1}, \ldots, z_{s}\right) Q^{\prime}$. Then $Q^{\prime} / \mathfrak{q}^{\prime} \simeq Q / \mathfrak{q}$. The ideals $q Q^{\prime}$ and $\left(z_{1}, \ldots, z_{s}\right) Q^{\prime}$ are analytically disjoint, whence, by [2], Theorem 3 ,

$$
e\left(\mathfrak{q}^{\prime}\right)=e\left(\mathfrak{q}^{\prime} /\left(z_{1}, \ldots, z_{s}\right) Q^{\prime}\right) \cdot e\left(\left(z_{1}, \ldots, z_{s}\right) Q^{\prime}\right)=e(\mathfrak{q})
$$

In a similar way we get $e\left(\mathfrak{m}^{\prime}\right)=e(\mathfrak{m})$.

After this change we have $\operatorname{dim} Q \geqslant s$.

(ii) Replace $Q$ by $Q^{\prime}=Q[z]_{\mathrm{m} Q[z]}$ where $z$ is an indeterminate over $Q$. Replace $m$ and $q$ by $m Q^{\prime}$ and $q Q^{\prime}$ respectively. Concerning the equations $e\left(m Q^{\prime}\right)=e(m)$ etc., cf. [5], 4.11. The ring $Q / \mathfrak{q}$ will undergo an alteration corresponding to a replacement of $K$ by $K(z)$ in (5.1). We achieve that $Q / \mathrm{m}$ is necessarily infinite.

(iii) Our next object is to achieve that $\operatorname{dim} Q=s$ and that $m$ has a reduction $\left(x_{1}, \ldots, x_{s}\right)$ such that $\theta\left(x_{i}\right)=X_{i}(i=1,2, \ldots, s)$. Put $\operatorname{dim} Q=t$. Note that, by (i), $t \geqslant s$. Put $q=\left(q_{1}, \ldots, q_{m}\right)$, and let $x_{1}, \ldots, x_{s}$ be elements of $Q$ such that $\theta\left(x_{i}\right)=$ $=X_{1}(i=1,2, \ldots, s)$. Then $\mathfrak{n}=\left(q_{1}, \ldots, q_{m}, x_{1}, \ldots, x_{s}\right)$. Consider the ideal generated by the $t$ elements

$$
a_{v 1} q_{1}+\cdots+a_{v m} q_{m}+b_{v 1} x_{1}+\cdots+b_{v s} x_{s} \quad(v=1,2, \ldots, t)
$$

where the $a_{\nu \mu}$ and the $b_{\nu \mu}$ belong to $Q$. This ideal will be a reduction of $\mathfrak{m}$ if and only if the residue classes of the $a_{v \mu}$ and $b_{\nu \mu}$ modulo $\mathfrak{m}$ do not belong to some proper algebraic set defined over $Q / \mathrm{m}$ ([7], Sections 5 and 6 ). Since, by (ii), $Q / \mathrm{m}$ is an infinite field, we can choose a system $\left\{a_{\nu \mu}, b_{\nu \mu}\right\}$ satisfying this condition and having the further property that the determinant $\left|b_{v \mu}\right|_{y, \mu-1.2 \ldots .}$ is a unit in $Q$. Thus, replacing the elements (5.2) by suitable linear combinations, we see that there is a reduction of $\mathrm{m}$ generated by $t$ elements of the form

$$
\begin{array}{ll}
c_{\nu 1} q_{1}+\cdots+c_{\nu m} q_{m}+x_{v} & (v=1,2, \ldots, s), \\
c_{v 1} q_{1}+\cdots+c_{v m} q_{m} & (\nu=s+1, \ldots, t) .
\end{array}
$$

We conclude that $t$ elements of this form will generate a reduction of $m$ whenever the residue classes of the $c_{\nu \mu}$ modulo $\mathrm{m}$ are outside some proper algebraic set. It follows that the $c_{\nu \mu}$ can be so determined that, if a denotes the ideal generated by the $t-s$ elements

$$
c_{p 1} q_{1}+\cdots+c_{\nu m} q_{m} \quad(\nu=s+1, \ldots, t),
$$

then $e(\mathfrak{m} / \mathfrak{a})=e(\mathfrak{m}), e(\mathfrak{q} / \mathfrak{a})=e(\mathfrak{q})$ (cf. Lemma 1; note that $s>0$ ). Thus we can replace $Q, \mathfrak{m}$, and $\mathfrak{q}$ by $Q / \mathfrak{a}, \mathfrak{m} / \mathfrak{a}$, and $\mathfrak{q} / \mathfrak{a}$. After this change $\operatorname{dim} Q=s$, and $m$ has a reduction generated by $s$ elements which are mapped on $X_{1}, \ldots, X_{s}$ by $\theta$, namely the residue classes modulo $a$ of the former elements

$$
c_{r 1} q_{1}+\cdots+c_{\nu m} q_{m}+x_{\nu} \quad(\nu=1,2, \ldots, s) .
$$


(iv) Replace $Q$ by its completion $Q^{\prime}$; replace $\mathfrak{m}$ and $q$ by $\mathfrak{m} Q^{\prime}$ and $q Q^{\prime}$ respectively. After this change $Q$ contains a field $k$ such that $\theta(k)=K$. For, by Geddes's method ([1], esp. Lemma 4, p. 338), a coefficient field of $Q / \mathfrak{q}$ can be lifted successively to a coefficient field of $Q / \mathfrak{q}^{2}, Q / \mathfrak{q}^{4}, \ldots, Q / \mathfrak{q}^{2^{n}}, \ldots, Q$.

$Q$ now has the dimension $s$ and contains a field $k$ such that $\theta(k)=K$. There is a reduction $\left(x_{1}, \ldots, x_{s}\right)$ of $\mathrm{m}$ such that $\theta\left(x_{i}\right)=X_{i}(i=1,2, \ldots, s)$.

Let $\varphi_{1}, \ldots, \varphi_{s}$ be the elements in $k\left[x_{1}, \ldots, x_{s}\right]$ obtained from $f_{1}, \ldots, f_{s}$ by replacing $X_{i}$ by $x_{i}(i=1,2, \ldots, s)$ and the coefficients in $K$ by their inverse images in $k$ under $\theta$. Then the ideal $\left(\varphi_{1}, \ldots, \varphi_{s}\right)$ in $Q$ is contained in $q$. We shall estimate the length of its $n^{\text {th }}$ power, $\left(\varphi_{1}, \ldots, \varphi_{s}\right)^{n}$, by applying the formula (1.2) with respect to the parameters $x_{1}, \ldots, x_{s}$. In view of the length-preserving properties of form ideals we readily see that

$$
L\left(I_{\nu}\left(\left(\varphi_{1}, \ldots, \varphi_{s}\right)^{n}\right)\right) \leqslant L\left(\left(f_{1}, \ldots, f_{s}\right)^{n}\right) \quad(v=0,1, \ldots . l-1) .
$$

Further, if $m$ is an integer such that

$$
\left(X_{1}, \ldots, X_{s}\right)^{m} \subseteq\left(f_{1}, \ldots, f_{s}\right),
$$

then

$$
\left(X_{1}, \ldots, X_{s}\right)^{m n} \subseteq I_{0}\left(\left(\varphi_{1}, \ldots, \varphi_{s}\right)^{n}\right) \quad(n=1,2,3, \ldots) .
$$

(Strictly speaking these two inclusions refer to different rings.) By means of an argument in [2] (lower half of p. 305) it follows that, for large values of $n$,

$$
L\left(I_{\nu}\left(\left(\varphi_{1}, \ldots, \varphi_{s}\right)^{n}\right)\right)=o\left(n^{s}\right) \text { if } I_{\nu}(0) \neq(0) .
$$

Inserting the estimates (5.3) and (5.4) in (1.2), and observing that, e.g. by Theorem 1,

$$
L\left(\left(f_{1}, \ldots, f_{s}\right)^{n}\right)=\left(\begin{array}{c}
n+s \\
s
\end{array}\right) L(q)
$$

we get

$$
L\left(\left(\varphi_{1}, \ldots, \varphi_{s}\right)^{n}\right)=\left(n^{s} / s !\right) L(q) e\left(x_{1}, \ldots, x_{s}\right)+o\left(n^{s}\right) .
$$

Hence, since $L\left(q^{n}\right) \leqslant L\left(\left(\varphi_{1}, \ldots, \varphi_{s}\right)^{n}\right)$ and $e\left(x_{1}, \ldots, x_{s}\right)=e(m)$,

$$
e(\mathfrak{q})=\lim _{n \rightarrow \infty} \frac{L\left(\mathfrak{q}^{n}\right)}{n^{s} / s !} \leqslant L(\mathfrak{q}) e(\mathrm{~m})
$$

which was to be proved.

As a corollary we obtain:

Let $\left(Q_{0}, Q\right)$ be a flat couple of local rings with maximal ideals $\left(\mathfrak{m}_{0}, \mathfrak{m}\right)$. Suppose that $Q / \mathrm{m}_{0} Q$ is isomorphic to a ring

$$
K\left[X_{1}, \ldots, X_{s}\right] /\left(f_{1}, \ldots, f_{s}\right),
$$

where $K$ is a field, $K\left[X_{1}, \ldots, X_{s}\right]$ a polynomial ring over $K$, and $\left(f_{1}, \ldots, f_{s}\right) a$ $\left(X_{1}, \ldots, X_{s}\right)$-primary ideal. In addition, suppose that $Q_{0}$ and $Q$ are equicharacteristic. Then

$$
e\left(Q_{0}\right) \leqslant e(Q) .
$$

For the proof, cf. the proof of the corollary of Theorem 3. 


\section{CH. LECH, Note on multiplicities of ideals}

Next we consider a type of residue class rings $Q / \mathfrak{q}$ for which the factor $d$ ! in Theorem 3 cannot be replaced by any smaller number. We shall prove the following statement:

Let $\left(Q_{0}, Q\right)$ be a flat couple of local rings with maximal ideals $\left(\mathfrak{m}_{0}, \mathfrak{m}\right)$. Suppose that there is a ring isomorphism

$$
Q / \mathfrak{m}_{0} Q \rightarrow K\left[X_{1}, \ldots, X_{s}\right] /\left(X_{1}, \ldots, X_{s}\right)^{m},
$$

where $K$ is a field and $K\left[X_{1}, \ldots, X_{s}\right]$ a polynomial ring over $K$. Then

$$
e\left(Q_{0}\right)=\frac{1}{L\left(\mathrm{~m}_{0} Q\right)} e(Q) .
$$

If $s=0$, then $m=m_{0} Q$, and the assertion is trivially true. Thus we can assume that $s>0$. We shall prove that

$$
\mathfrak{m}^{m}=\mathfrak{m}_{0} \mathfrak{m}^{m-1}
$$

which means that $m_{0} Q$ is a reduction of $\mathfrak{m}$ (cf. [7]). This implies that

$$
e(\mathrm{~m})=e\left(\mathrm{~m}_{0} Q\right)=e\left(\mathrm{~m}_{0}\right) L\left(\mathrm{~m}_{0} Q\right)
$$

(cf. the appendix), and thus gives the result.

Both sides of (5.6) contain the ideal $\mathrm{m}_{0}^{m} Q$. By passing, if necessary, from $\left(Q_{0}, Q\right)$ to $\left(Q_{0} / \mathrm{m}_{0}^{m}, Q / \mathrm{m}_{0}^{m} Q\right)$ we can assume that $\mathrm{m}_{0}^{m}=(0)$ (cf. the appendix). Then $Q_{0}$ and $Q$ are zero-dimensional.

Let $\varkappa_{\iota}(\iota \in S)$ be elements in $Q$ representing a basis of $Q / \mathrm{m}$ over $Q_{0} / \mathrm{m}_{0}$. Let $x_{1}, \ldots, x_{s}$ be representatives in $Q$ of those elements in $Q / \mathfrak{m}_{0} Q$ which correspond to $X_{1}, \ldots, X_{s}$ under the isomorphism (5.5). If $\sigma=\left(\sigma_{1}, \ldots, \sigma_{s}\right)$ is a sequence of $s$ non-negative integers, we shall write $x^{\sigma}$ for $x_{1}^{\sigma_{1}} \ldots x_{s}^{\sigma_{s}}$. If $\sigma=\left(\sigma_{1}, \ldots, \sigma_{s}\right)$ is an arbitrary sequence of $s$ integers, we put $|\sigma|=\sigma_{1}+\cdots+\sigma_{s}$ and write $\sigma \geqslant 0$ if $\sigma_{i} \geqslant 0(i=1,2, \ldots, s)$; we define $\sigma+\tau$ as $\left(\sigma_{1}+\tau_{1}, \ldots, \sigma_{s}+\tau_{s}\right)$ if $\tau=\left(\tau_{1}, \ldots, \tau_{s}\right)$. When we use the symbol $x^{\sigma}$ it will always be understood that $\sigma \geqslant 0$.

The elements $\varkappa_{\iota} x^{\tau}(\iota \in S,|\tau|<m)$ represent a basis of $Q / \mathrm{m}_{0} Q$ over $Q_{0} / \mathfrak{m}_{0}$. For, in view of $(5.5)$,

$$
Q \subseteq \sum_{\imath} Q_{0} x_{t}+\left(x_{1}, \ldots, x_{s}\right) Q+m_{0} Q
$$

where $\sum_{c} Q_{0} x_{t}$ stands for the $Q_{0}$-submodule of $Q$ generated by the $\varkappa_{\iota}$. Iterating this inclusion and observing that $\left(x_{1}, \ldots, x_{s}\right)^{m} Q \subseteq \mathfrak{m}_{0} Q$, we get

$$
Q \subseteq \sum_{\substack{i \\|x|<m}} Q_{0} \varkappa_{\imath} x^{\tau}+\mathfrak{m}_{0} Q
$$

On the other hand, if there were a relation

$$
\sum_{\substack{i \\|\tau|<m}} a_{\imath, \tau} \varkappa_{\iota} x^{\tau} \in \mathfrak{M}_{0} Q \quad\left(a_{t, \tau} \in Q_{0}\right)
$$


with $a_{t, \tau}=0$ for all but a finite number of indices $(\iota, \tau)$ and with at least one $a_{t, \tau} \notin \mathrm{m}_{0}$, one could assume (after a possible multiplication with some $x^{\sigma}$ ) that the range of $\tau$ were just $|\tau|=m-1$, and readily get a contradiction to the isomorphism (5.5).

As is shown in the appendix, it follows that the elements $\varkappa_{t} x^{\tau}(\iota \in S,|\tau|<m)$ also form a basis of $Q$ over $Q_{0}$ (because these rings are zero-dimensional). Consequently, denoting by $M$ the $Q_{0}$-submodule of $Q$ generated by the $\varkappa_{t}$, there are for each $y \in Q$ unique coefficients $a(y ; \tau) \in M(\tau \geqslant 0,|\tau|<m)$ such that

$$
y=\sum_{|\tau|<m} a(y ; \tau) x^{\tau}
$$

We define $a(y ; \tau)$ as $0(\epsilon M)$ when $\tau \neq 0$ or $|\tau| \geqslant m$. Further, if $a, b \in M$ and $a-b \in \mathrm{m}_{0}^{v} M$, we shall write $a \equiv b\left(\mathfrak{m}_{0}^{v} M\right)(y=0,1,2, \ldots)$.

Denote by $\mathfrak{a}$ the ideal $\left(x_{1}, \ldots, x_{s}\right)$ in $Q$. Then $\mathfrak{m}=\mathfrak{m}_{0} Q+\mathfrak{a}$. To prove (5.6) it suffices to show that

$$
\mathfrak{a}^{m} \subseteq \sum_{\nu=0}^{m-1} \mathfrak{m}_{0}^{m-\nu} \mathfrak{a}^{v}
$$

To do this we introduce an integralvalued, positive, non-increasing function $\varphi(v)$, defined for $v=0,1, \ldots, m-1$, which we assume to have the property that

$$
\mathfrak{a}^{m} \subseteq \sum_{\nu=0}^{m-1} \mathfrak{m}_{0}^{\varphi(v)} \mathfrak{a}^{\nu}
$$

We shall show quite generally how this function can be improved upon. Our result will make it possible to pass from $\varphi(\nu) \equiv 1$ to $\varphi(\nu) \equiv m-\nu$. This will complete our proof, for, in view of the fact that $\mathfrak{a}^{m} \subseteq \mathrm{m}_{0} Q=\mathfrak{m}_{0} \mathfrak{a}^{0}$, it is evident that the function $\varphi(v) \equiv 1$ satisfies all our assumptions.

First we prove that, for $k=0,1,2, \ldots ; 0 \leqslant|\tau| \leqslant m-1$,

$$
y \in \mathfrak{m}_{0}^{k} \mathfrak{a}^{m} \text { implies } a(y ; \tau) \in \mathfrak{m}_{0}^{\varphi(|\tau|)+k} M .
$$

This is true for $k \geqslant m$, since then $y=0$. We proceed by induction on $k$. Assume that $0 \leqslant k<m$, and that (5.9) has been proved for all larger values of the inductive variable. From (5.8) and the fact that every element in $Q$ can be written on the form (5.7), it follows that $y$ can be written as a sum of terms of the form $a x^{\tau^{\prime}} x^{\tau^{\prime \prime}}$ where $\left|\tau^{\prime}\right|<m,\left|\tau^{\prime \prime}\right|<m$, and $a \in \mathfrak{m}_{0}^{\psi\left(\left|\tau^{\prime}\right|\right)+k} M$. It suffices to prove the conclusion of (5.9) for each such term. When $\left|\tau^{\prime}\right|+\left|\tau^{\prime \prime}\right|<m$, we get the result directly from the fact that $\varphi\left(\left|\tau^{\prime}\right|+\left|\tau^{\prime \prime}\right|\right) \leqslant \varphi\left(\left|\tau^{\prime}\right|\right)$. When $\left|\tau^{\prime}\right|+\left|\tau^{\prime \prime}\right| \geqslant m$, then, since $\varphi\left(\left|\tau^{\prime}\right|\right)>0$, we have $a x^{\tau^{\prime}} x^{\tau^{\prime \prime}} \in \mathrm{m}_{0}^{k+1} \mathrm{a}^{m}$, and the result follows by the inductive hypothesis.

For $i=1,2, \ldots, s$, let $\varepsilon_{i}$ denote the sequence $(0, \ldots, 1, \ldots, 0)$ whose $i^{\text {th }}$ element is 1 and whose remaining elements are 0 . We shall prove that, if

$$
|\sigma|=m, \sigma \geqslant 0, \sigma+\varepsilon_{i}-\varepsilon_{j} \geqslant 0,0 \leqslant|\tau| \leqslant m-2,
$$

then

$$
a\left(x^{\sigma} ; \tau\right) \equiv a\left(x^{\sigma+\varepsilon_{i}} ; \tau+\varepsilon_{i}\right) \equiv a\left(x^{\sigma+\varepsilon_{i}-\varepsilon_{j}} ; \tau+\varepsilon_{i}-\varepsilon_{j}\right) \quad\left(\mathrm{m}_{0}^{\sigma(|\tau|+1)+1} M\right)
$$


The left and the right congruence are quite analogous. Thus it suffices to prove the left one. If $\tau+\varepsilon_{i} \neq 0$, then $\tau \neq 0$, and the result follows from the fact that we have defined $a(y ; \tau)$ as 0 when $\tau \neq 0$. Assume then that $\tau+\varepsilon_{i} \geqslant 0$. Expressing $x^{\sigma}$ on the form (5.7) and multiplying by $x_{i}$, we get

$$
x^{\sigma+\varepsilon_{i}}=\sum_{\tau} a\left(x^{\sigma} ; \tau\right) x^{\tau+\varepsilon_{i}}
$$

By (5.9) and the fact that the function $\varphi(v)$ is positive, the coefficients $a\left(x^{\sigma} ; \tau\right)$ that occur here, belong to $m_{0} M$. Thus the terms with $|\tau|=m-1$ belong to $m_{0} \mathfrak{a}^{m}$. The result therefore follows from (5.9).

By repeated application of the congruence

$$
a\left(x^{\sigma} ; \tau\right) \equiv a\left(x^{\sigma+\varepsilon_{i}-\varepsilon_{j}} ; \tau+\varepsilon_{i}-\varepsilon_{j}\right)\left(\mathrm{m}_{0}^{\varphi(|\tau|+1)+1} M\right)
$$

we see that, if

$$
|\sigma|=\left|\sigma^{\prime}\right|=m, \sigma \geqslant 0, \sigma^{\prime} \geqslant 0,0 \leqslant|\tau| \leqslant m-2,
$$

then

$$
a\left(x^{\sigma} ; \tau\right) \equiv a\left(x^{\sigma^{\prime}} ; \tau+\sigma^{\prime}-\sigma\right) \quad\left(\mathfrak{m}_{0}^{\phi(\{\tau \mid+1)+1} M\right) .
$$

Since $\tau-\sigma \neq 0$, it is always possible to choose $\sigma^{\prime}$ such that $\tau+\sigma^{\prime}-\sigma \neq 0$. Hence, for $|\sigma|=m, 0 \leqslant|\tau| \leqslant m-2$,

$$
a\left(x^{\sigma} ; \tau\right) \equiv 0 \quad\left(\mathfrak{m}_{0}^{\varphi(|\tau|+1)+1} M\right) .
$$

In view of (5.7) and the fact that $a=\left(x_{1}, \ldots, x_{s}\right)$, this means that the function $\psi(v)$ defined as $\varphi(v+1)+1$ for $v=0,1, \ldots, m-2$ and as $\varphi(m-1)$ for $\nu=m-1$ will have all the properties which we have assumed for $\varphi(v)$. If $\varphi(\nu) \equiv \min (m-\nu, k)$, then $\psi(\nu) \equiv \min (m-\nu, k+1)(1 \leqslant k \leqslant m-1)$. Thus we can pass from $\varphi(v) \equiv \mathbf{l} \equiv \min (m-v, 1)$ to $\varphi(v) \equiv m-v \equiv \min (m-v, m)$. This completes our proof.

The results of this section, together with Theorem 4, strongly suggest that, for $d>1$, the factor $d$ ! in the Corollary of Theorem 3 could be replaced by a smaller number. I hazard the conjecture that this factor can actually be replaced by 1 , i.e. that

$$
e\left(Q_{0}\right) \leqslant e(Q)
$$

for flat couples $\left(Q_{0}, Q\right)$ of local rings of the same dimension. (This would imply that $e\left(Q_{0}\right) \leqslant e(Q)$ for all flat couples $\left(Q_{0}, Q\right)$ of local rings, whether of the same dimension or not.) 


\section{APPENDIX}

Let $R$ be a commutative ring with unity element, and let $M$ be a unitary $\boldsymbol{R}$-module. We have the following definition by Serre:

$M$ is called $R$-flat (or flat) if the functor

$$
T: T(E)=E \otimes_{R} M
$$

defined on the categary of unitary $R$-modules, is exact.

If $M$ is flat, it satisfies in particular the condition that, for every ideal $\mathfrak{a}$ of $R$, the sequence

$$
0 \rightarrow \mathfrak{a} \otimes_{R} M \rightarrow R \otimes_{R} M
$$

is exact. Using the functor Tor, Serre shows that this condition is also sufficient ([10], p. 35). We shall derive another criterion, namely:

$M$ is flat if and only if $(\mathfrak{a}: \alpha) M=\mathfrak{a} M: \alpha$ for every ideal $\mathfrak{a}$ and every element $\alpha$ in $R$.

Proof. 1) Suppose that $(\mathfrak{a}: \alpha) M=\mathfrak{a} M: \alpha$ for all $\mathfrak{a}, \alpha$. We have to show that for any fixed $a$ the sequence $0 \rightarrow \mathfrak{a} \otimes_{R} M \rightarrow R \otimes_{R} M$ is exact. In other words, if $\alpha_{1}, \ldots, \alpha_{r}$ are elements of $\mathfrak{a}$ and $\gamma_{1}, \ldots, \gamma_{r}$ elements of $M$ such that $\sum_{\nu=1}^{r} \alpha_{\nu} \gamma_{\nu}=0$, then we must show that $\sum_{\nu=1}^{r} \alpha_{\nu} \otimes \gamma_{\nu}=0$ in $a \otimes_{R} M$. This will be done by induction on $r$. Assume that $r \geqslant 2$ and that the assertion is true for all smaller values of the inductive variable. Since $\sum_{v=1}^{r} \alpha_{\nu} \gamma_{\nu}=0$, we have $\alpha_{r} \gamma_{r} \in\left(\alpha_{1}, \ldots, \alpha_{r-1}\right) M$. Hence, by our assumption, $\gamma_{r} \in\left(\left(\alpha_{1}, \ldots, \alpha_{r-1}\right): \alpha_{r}\right) M$. Let $\beta_{1}, \ldots, \beta_{s}$ be elements of $\left(\alpha_{1}, \ldots, \alpha_{r-1}\right): \alpha_{r}$ and $\gamma_{1}^{\prime}, \ldots, \gamma_{s}^{\prime}$ elements of $M$ such that $\gamma_{r}=\sum_{\mu=1}^{s} \beta_{\mu} \gamma_{\mu}^{\prime}$. Then $\alpha_{r} \otimes \gamma_{r}=\sum_{\mu=1}^{s} \alpha_{r} \otimes \beta_{\mu} \gamma_{\mu}^{\prime}=\sum_{\mu=1}^{s} \alpha_{r} \beta_{\mu} \otimes \gamma_{\mu}$. Since $\alpha_{r} \beta_{\mu} \in\left(\alpha_{1}, \ldots, \alpha_{r-1}\right)$, this means that we can write $\alpha_{r} \otimes \gamma_{r}$ on the form $\sum_{\nu=1}^{r-1} \alpha_{\nu} \otimes \gamma_{\nu}^{\prime \prime}$ with $\gamma_{\nu}^{\prime \prime} \in M$. Then $\sum_{\nu=1}^{r} \alpha_{\nu} \otimes \gamma_{\nu}=$ $=\sum_{\nu=1}^{r-1} \alpha_{\nu} \otimes\left(\gamma_{\nu}+\gamma_{\nu}^{\prime \prime}\right)$. As this relation in $\mathfrak{a} \otimes_{R} M$ obviously implies the relation $0=\sum_{\nu=1}^{r} \alpha_{\nu} \gamma_{\nu}=\sum_{\nu=1}^{r-1} \alpha_{\nu}\left(\gamma_{\nu}+\gamma_{\nu}^{\prime \prime}\right)$ in $R \otimes_{R} M$, it follows by the inductive hypothesis that $\sum_{v=1}^{r} \alpha_{\nu} \otimes \gamma_{\nu}=0$. Thus we have reduced the proof to the case $r=1$. In this case we can proceed in the same way as above, interpreting $\left(\alpha_{1}, \ldots, \alpha_{r-1}\right)$ as the zero ideal. We get $\alpha_{1} \otimes \gamma_{1}=\sum_{\mu=1}^{s} \alpha_{1} \beta_{\mu} \otimes \gamma_{\mu}$ with $\alpha_{1} \beta_{\mu}=0(\mu=1,2, \ldots, s)$, i.e. $\alpha_{1} \otimes \gamma_{1}=0$, which was to be proved. 
2) Suppose that $M$ is flat, and let us show that $(\mathfrak{a}: \alpha) M=\mathfrak{a} M: \alpha$. We shall need two auxiliary statements.

(i) Suppose that $E$ and $F$ are two unitary $R$-modules and that $F \subseteq E$. By applying the functor $T$ defined above to the exact sequence

$$
0 \rightarrow F \rightarrow E \rightarrow E / F \rightarrow 0,
$$

we obtain a canonical isomorphism

$$
(E / F) \otimes_{R} M \simeq E \otimes_{R} M / F \otimes_{R} M .
$$

(ii) Similarly, applying $T$ to the sequence

$$
0 \rightarrow \mathfrak{a} \rightarrow R,
$$

we obtain a canonical isomorphism

$$
\mathfrak{a} \otimes_{R} M \simeq \mathfrak{a} M .
$$

Now, let $a$ be an arbitrary ideal and $\alpha$ an arbitrary element in $R$. Consider the exact sequence

$$
(\mathfrak{a}: \alpha) / \mathfrak{a} \rightarrow R / \mathfrak{\alpha} \rightarrow R / \mathfrak{a},
$$

where the first map is induced by inclusion and the second by multiplication with $\alpha$. Applying $T$ to this sequence and taking the above canonical isomorphisms into account, we get a sequence

$$
(\mathfrak{a}: \alpha) M / \mathfrak{a} M \rightarrow M / \mathfrak{a} M \stackrel{\alpha}{\rightarrow} M / \mathfrak{a} M,
$$

whose exactness implies that

$$
(\mathfrak{a}: \alpha) M / \mathfrak{a} M=\mathfrak{a} M: \alpha / \mathfrak{a} M,
$$

i.e.

$$
(\mathfrak{a}: \alpha) M=\mathfrak{a} M: \propto,
$$

which was to be proved.

The relation $(\mathfrak{a}: \alpha) M=\mathfrak{a} M: \alpha$ is well known from the special situation where $R$ is a local ring and $M$ its completion (cf. [9], p. 9; ef. also [10], Prop. 27, p. 39). Moreover, there is a result by Samuel-Nagata to the effect that, if the condition $(\mathfrak{a}: \alpha) M=\mathfrak{a} M: \alpha$ is satisfied for all $\mathfrak{a}$ and $\alpha$ in $R$, then

$$
(\mathfrak{a} \cap \mathfrak{b}) M=\mathfrak{a} M \cap \mathfrak{b} M
$$

for all ideals $\mathfrak{a}$ and $\mathfrak{b}$ of $R$. (The result is formulated for the special situation mentioned above, but the proof extends to the general case. See [3].)

Flat couples (of rings) can be defined as follows (Serre [10], Def. 4 and Prop. 22, p. 36):

Let $R$ be a commutative ring and $R_{0}$ a subring of $R$. Assume that $R$ and $R_{0}$ have a common unity element. The couple $\left(R_{0}, R\right)$ is called flat if $R$ is $R_{0}$-flat and if, for every ideal $\mathfrak{a}_{0}$ of $R_{0}$, we have $\mathfrak{a}_{0} R \cap R_{0}=\mathfrak{a}_{0}$. 
It suffices to require that $\mathfrak{a}_{0} R \cap R_{0}=a_{0}$ for all maximal ideals of $R_{0}$. For suppose that $a_{0}$ is an ideal and $\alpha_{0}$ an element of $R_{0}$ such that $\alpha_{0} \in \mathfrak{a}_{0} R \cap R_{0}$, $\alpha_{0} \notin \mathfrak{a}_{0}$. Then $l \in \mathfrak{a}_{0} R: \alpha_{0}=\left(\mathfrak{a}_{0}: \alpha_{0}\right) R$. Thus, if $\mathfrak{m}_{0}$ is a maximal ideal containing $\mathfrak{a}_{0}: \alpha_{0}$, we get $\mathfrak{m}_{0} R \cap R_{0}=R_{0} \neq \mathfrak{m}_{0}$.

We now record those facts about flatness that are used in the present note.

Let the module $M$ be $R$-flat. Suppose that $\mathfrak{a}$ is an ideal and $S$ a multiplicatively closed set in $R$. Then $M / \mathfrak{a} M$ is $(R / \mathfrak{a})$-flat, and $M_{S}$ is $R$-flat (cf. [10], or apply the criterion a $M: \alpha=(\mathfrak{a}: \alpha) M)$.

Let $\left(Q_{0}, Q\right)$ be a flat couple of local rings with maximal ideals $\left(\mathfrak{m}_{0}, \mathfrak{m}\right)$. This means that $Q$ is $Q_{0}$-flat and that $\mathrm{m}_{0} Q \subseteq \mathrm{m}$. It follows that, if $\mathrm{a}_{0}$ is a proper ideal of $Q_{0}$, and if $\mathfrak{p}$ is a prime ideal of $Q$ containing $\mathfrak{m}_{0}$, then the couples $\left(Q_{0} / \mathrm{a}_{0}, Q / \mathfrak{a}_{0} Q\right)$ and $\left(Q_{0}, Q_{\mathfrak{p}}\right)$ are flat (cf. the preceding paragraph).

If $\mathfrak{a}_{0}$ is an ideal of $Q_{0}$, then $\mathfrak{a}_{0} Q / \mathfrak{a}_{0} \mathfrak{m}_{0} Q$ is a free $\left(Q / \mathfrak{m}_{0} Q\right)$-module, and if $\alpha_{1}, \ldots, \alpha_{n}$ form a minimal basis of $\mathfrak{a}_{0}$, they represent a basis of $\mathfrak{a}_{0} Q / \mathfrak{a}_{0} \mathfrak{m}_{0} Q$ over $Q / \mathrm{m}_{0} Q$. For suppose that $\gamma_{1}, \ldots, \gamma_{n}$ are elements of $Q$ such that

$$
\alpha_{1} \gamma_{1}+\cdots+\alpha_{n} \gamma_{n}=0
$$

Then

$$
\gamma_{n} \in\left(\alpha_{1}, \ldots, \alpha_{n-1}\right) Q: \alpha_{n}=\left(\left(\alpha_{1}, \ldots, \alpha_{n-1}\right): \alpha_{n}\right) Q \subseteq \mathfrak{m}_{0} Q
$$

and similarly $\gamma_{1}, \ldots, \gamma_{n-1} \in m_{0} Q$. This gives the result.

Suppose that $\mathfrak{m}_{0} Q$ is $\mathfrak{m}$-primary. Since $\mathfrak{m}_{0}^{v} Q / \mathfrak{m}_{0}^{\nu+1} Q$ is a free $\left(Q / \mathfrak{m}_{0} Q\right)$-module with a basis of $L\left(\mathfrak{m}_{0}^{v+1}\right)-L\left(\mathfrak{m}_{0}^{v}\right)$ elements, we have

$$
L\left(\mathfrak{m}_{0}^{v+1} Q\right)-L\left(\mathrm{~m}_{0}^{v} Q\right)=\left(L\left(\mathfrak{m}_{0}^{v+1}\right)-L\left(\mathfrak{m}_{0}^{v}\right)\right) L\left(\mathrm{~m}_{0} Q\right),
$$

hence

$$
L\left(\mathfrak{m}_{0}^{v} Q\right)=L\left(\mathfrak{m}_{0}^{v}\right) L\left(\mathfrak{m}_{0} Q\right) \quad(v=1,2,3, \ldots) .
$$

It follows that

$$
\begin{aligned}
& \operatorname{dim} Q_{0}=\operatorname{dim} Q \\
& e\left(\mathfrak{m}_{0} Q\right)=e\left(\mathrm{~m}_{0}\right) L\left(\mathfrak{m}_{0} Q\right)
\end{aligned}
$$

(cf. the definition of multiplicity).

If $\mathfrak{m}_{0} Q$ is not $\mathfrak{m}$-primary, it has a minimal prime ideal $\mathfrak{p} \neq \mathfrak{m}$. Since $\left(Q_{0}, Q_{\mathfrak{p}}\right)$ is flat and since $\mathfrak{m}_{0} Q_{\mathfrak{p}}$ is a $\left(p Q_{\mathfrak{p}}\right)$-primary ideal, we deduce that $\operatorname{dim} Q_{0}=\operatorname{dim} Q_{\mathfrak{p}}$, hence that $\operatorname{dim} Q_{0}<\operatorname{dim} Q$. Thus it is equivalent to say that $\mathfrak{m}_{0} Q$ is $\mathfrak{m}$-primary and that $Q_{0}$ and $Q$ have the same dimension.

Assume that $Q_{0}$ and $Q$ are zero-dimensional. Then $Q$ is a free $Q_{0}$-module, and if $\omega_{\iota}(\iota \in S)$ are representatives in $Q$ of a basis of $Q / \mathfrak{m}_{0} Q$ over $Q_{0} / \mathfrak{m}_{0}$, then the $\omega_{\imath}$ form a basis of $Q$ over $Q_{0}$. To see this, we first observe that

$$
Q \subseteq \sum_{\iota} Q_{0} \omega_{\iota}+\mathfrak{m}_{0} Q
$$

where $\sum_{\imath} Q_{0} \omega_{\imath}$ denotes the $Q_{0}$-submodule of $Q$ generated by the $\omega_{\imath}$. Iterating this inclusion, we obtain

$$
Q \subseteq \sum_{\iota} Q_{0} \omega_{\imath}+\mathfrak{m}_{0}^{\prime} Q \quad(\nu=1,2,3, \ldots) .
$$




\section{CH. LECH, Note on multiplicities of ideals}

Because $\mathfrak{m}_{0}^{v}=(0)$ for $\nu$ sufficiently large, this means that $Q \subseteq \sum_{\imath} Q_{0} \omega_{\imath}$. It remains to show that the $\omega_{\imath}$ are independent. Assume that there is a relation

$$
\alpha_{1} \omega_{1}+\cdots+\alpha_{n} \omega_{n}=0
$$

with $\alpha_{\nu} \in Q_{0}, \omega_{\nu} \in\left\{\omega_{\iota} \mid \iota \in S\right\}$. Let $\alpha_{1}, \ldots, \alpha_{r}$ be a minimal basis of the ideal $\left(\alpha_{1}, \ldots, \alpha_{n}\right)$ in $Q_{0}$. Then there are relations

$$
\alpha_{\nu}=\sum_{\mu=1}^{r} \beta_{v, \mu} \alpha_{\mu} \quad(\nu=r+1, \ldots, n)
$$

with $\beta_{v, \mu} \in Q_{0}$. Inserting these in $\left({ }^{*}\right)$, we get

$$
\alpha_{1}\left(\omega_{1}+\beta_{r+1,1} \omega_{r+1}+\cdots+\beta_{n, 1} \omega_{n}\right)+\cdots+\alpha_{r}\left(\omega_{r}+\cdots\right)=0
$$

Hence

$$
\omega_{1}+\beta_{r+1,1} \omega_{r+1}+\cdots+\beta_{n, 1} \omega_{n} \in\left(\left(\alpha_{2}, \ldots, \alpha_{r}\right): \alpha_{1}\right) Q \subseteq \mathrm{m}_{0} Q,
$$

which is a contradiction, as the $\omega_{\iota}$ represent a basis of $Q / \mathfrak{m}_{0} Q$ over $Q_{0} / \mathfrak{m}_{0}$.

\section{R E F E R E N C E S}

1. Geddes, A., A short proof of the existence of coefficient fields for complete, equicharacteristic local rings. J. London Math. Soc. 29 (1954), 334-341 .

2. LeCh, C., On the associativity formula for multiplicities. Ark. Mat. 3 (1958), 301-314 (1956).

3. Nagata, M., Some remarks on local rings. Nagoya Math. J. 6 (1953), 53-58.

4. - The theory of multiplicity in general local rings. Proceedings of the international symposium on algebraic number theory. Tokyo-Nikko, 1955, pp. 191-226. Science Cuuncil of Japan, Tokyo, 1956.

5. NorthcotT, D. G., Ideal theory. Cambridge Tracts in Math. and Math. Phys. 42. Cambridge 1953, $111 \mathrm{pp}$.

6. - Semi-regular local rings. Mathematika 3 (1956), 117-126.

7. Northcott, D. G. and Rees, D., Reductions of ideals in local rings. Proc. Cambridge Phil. Soc. 50 (1954), 145-158.

8. Rees, D., The grade of an ideal or module. Proc. Cambridge Phil. Soc. 53 (1957), $28-42$.

9. Samuel, P., Algèbre locale, Mémorial Sci. Math. 123. París 1953. 76 pp.

10. Serre, J.-P., Géométrie algébrique et géométrie analytique. Ann. Inst. Fourier, Grenoble 6 (1955-1956), 1-42. 